\title{
Dynamic heterogeneity in random and gradient copolymers: A computational investigation
}

Mohammed Zakaria Slimani, ${ }^{1}$ Angel J. Moreno, ${ }^{2, *}$ Giulia Rossi, ${ }^{3}$ and Juan Colmenero ${ }^{1,2,4}$

${ }^{1}$ Donostia International Physics Center, Paseo Manuel de Lardizabal 4, 20018 San Sebastián, Spain.

${ }^{2}$ Centro de Física de Materiales (CSIC, UPV/EHU) and

Materials Physics Center MPC, Paseo Manuel de Lardizabal 5, 20018 San Sebastián, Spain.

${ }^{3}$ INSERM UMR-S 665, DSIMB 6, rue Alexandre Cabanel, 75739 Paris Cedex 15, France.

${ }_{4}^{4}$ Departamento de Física de Materiales, Universidad del País Vasco

(UPV/EHU), Apartado 1072, 20080 San Sebastián, Spain.

By means of molecular dynamics simulations, we investigate the structural relaxation in disordered random copolymers and lamellar phases of gradient copolymers, containing chemical species of very different mobilities. Two models have been investigated: a generic bead-spring system and a MARTINI coarse-grained model of a polyester resin. The lamellar phase of the gradient copolymer is formed by domains rich in one species and poor in the other one, which are separated by broad interfaces. Unlike in strongly segregated block copolymers, there is a finite probability of finding monomers of a given species at any position within the domains rich in the other species. A direct consequence of this feature is that monomers can probe very different chemical environments, and because of the strong dynamic asymmetry between the two components, their relaxation are characterized by an extreme dynamic heterogeneity. This is confirmed by an analysis of dynamic correlators as a function of the distance to the interface. In the case of random copolymers longrange ordering is not possible, and local microsegregation results in a much weaker dynamic heterogeneity. The former features are consistent with the experimental observation of narrow glass transitions in random copolymers but extremely broad ones in lamellar gradient copolymers.

*Corresponding author:wabmosea@ehu.es 


\section{INTRODUCTION}

The existence of permanent chemical links between different types of monomers (A and B) prevents macrophase separation in binary copolymers. However microsegregation occurs. This phenomenon is favoured by increasing the chain length and the energetic incompatibility of the two species. For large values of these control parameters segregation leads, for diblock copolymers, to long-ranged ordered mesophases, with well-defined pure domains separated by narrow interfaces. Different ordered morphologies can be obtained by varying the chain composition. These include, from asymmetric to symmetric compositions, spherical, cylinder, gyroid and lamellar phases [1-4].

Gradient copolymers are a particular case of binary copolymers, in which the population of each species exhibits a monotonous gradual change from one side of the chain to the other [5-10], instead of the step-like composition of diblock copolymers. Theory [11-15], computer simulations [16] and experiments [17-27] have shown that gradient copolymers can also exhibit long-range ordered phases even for smooth composition profiles. The domains are separated by broad smooth interfaces with a strong degree of A-B mixing. Unlike in strongly segregated phases of diblock copolymers, there is a significant probability of finding a monomer of a given species at any distance from the interface, even deep inside a domain rich in the other species. The chains cannot adopt conformations that fully prevent A-B contacts over long distances. This is a direct consequence of the many different realizations, originating from the gradient-like distribution, of the composition patterns along the backbones of all chains in the system.

Polymers are glass-formers [28]. On decreasing temperature the structural $\alpha$-relaxation time (characterizing segmental dynamics) exhibits a dramatic increase by about 14 decades from the picosecond scale up to 100 seconds (the latter time scale being often used to define the laboratory glass transition temperature $T_{\mathrm{g}}$ ). The relaxation scenario of the homopolymer is modified when it is linked to a block of very different mobility (very different $T_{\mathrm{g}}$ ). A general observation in ordered phases of diblock copolymers is that the $\alpha$-relaxation times of the blocks are close to those of the corresponding homopolymers, with shifts to shorter or longer times if the other block has respectively a higher or lower mobility [26, 29-32]. In spite of the similar values of the $\alpha$-time scales, the dynamic response exhibits a strong broadening in the low-frequency side in comparison with the corresponding homopolymer [26, 29-32]. 
This feature is usually rationalized in terms of dynamic heterogeneity. It is assumed that the segmental relaxation in the center of the domains is the same as in the corresponding homopolymer, but it is strongly perturbed as the interface is approached, leading to gradients of mobility . Recent simulations of lamellar, cylinder and spherical phases of bead-spring diblock copolymers are consistent with this picture $[33,34]$. The simulations also suggest that the gradients of mobility essentially arise as an interfacial effect, the homopolymer dynamics being recovered at about 2-4 nanometers from the interfacial plane.

A much more complex situation may be expected in the case of ordered mesophases of gradient copolymers. As mentioned above, domains are separated by broad interfaces with a high degree of A-B mixing, and moreover there is a significant population of both species in the two kinds of domains (A-rich and B-rich), even at long distances from the interface. Therefore, one might expect that the global dynamic response originates from contributions with very different time scales, because of the rather different chemical environments that can be probed by monomers of a same species. According to their specific position in the interfacial region or in the domains, monomers can probe environments with similar concentration of both species or can be mostly surrounded by monomers of their same or the other species. Because of the different intrinsic mobilities of both species, such different environments can lead to extreme dynamic heterogeneity. Unlike in the case of strongly segregated diblock copolymers, where gradients of mobility are essentially an interfacial effect, in gradient copolymers dynamic heterogeneities are expected to extend over much larger length scales. Even it might happen that the homopolymer dynamics is not recovered in any region of the domains. The existence of an extreme dynamic heterogeneity is also suggested by differential scanning calorimetry, which reveals a glass transition occurring over an extremely broad temperature interval $[10,17,19,20,23,24,26,35]$. For this reason gradient copolymers have been proposed as excellent materials for shock absorption and vibration damping [23]. The former observation is rather different from that in ordered phases of diblock copolymers, which exhibit a double glass transition [10, 19, 20, 24], reflecting the separation in the time scales of the two blocks.

Most of the investigations on gradient copolymers have focused on their structural, thermodynamic and mechanical properties. Only a few works have investigated their segmental relaxation $[16,26]$. It has been found that, for the same temperature and concentration, the global response of a component in the lamellar gradient copolymer is characterized by 
relaxation times that are intermediate between those in the purely random copolymer, and those in the lamellar diblock copolymer $[16,26]$. However, solving the different contributions to dynamic heterogeneity from the experimental spectra, which provide a global response, and the interpretation of such global results, is an extremely complex problem. Unlike in simple diblock copolymers [36], in gradient copolymers it is very difficult, or even impossible, to implement selective methods as, e.g., isotopic labelling of a specific sector of the chain backbone. This is due to the stochastic character, following a gradient probability distribution, of the compositional pattern along the chain backbone.

With these ideas in mind, in this article we present large-scale molecular dynamics (MD) simulations of lamellar phases of gradient copolymers, in order to provide valuable microscopic information for the interpretation of experimental results. Due to the typical spatial dimensions of the domains (several tens of nanometers), simulations of slow dynamics in such phases with fully atomistic force fields is far beyond the present computational capabilities. However such scales can be explored by coarse-grained models. Specifically, we have used a generic bead-spring model [37] and a realistic coarse-grained model of a common polyester resin. For the latter we have used the MARTINI force field, within the coarse-graining scheme proposed by Rossi et al. in Ref. [38]. Initially developed for lipids and surfactants $[39,40]$, MARTINI has been extended to other systems including proteins $[41,42]$ and common polymers [43-47]. Likewise, we have performed MD simulations of random copolymers with the same macroscopic concentration as the gradient copolymers, by using the same bead-spring and MARTINI models. Because of the random compositional pattern along the backbone, purely random copolymers cannot be arranged to form ordered mesophases, but still can exhibit some degree of microsegregation in order to minimize A-B contacts [48-53]. Therefore, the variety of local environments that can be probed by the monomers in random copolymers is strongly reduced in comparison with the case of gradient copolymers. For this reason, a strong reduction of the dynamic heterogeneity is also expected. Indeed a narrow glass transition is found in calorimetric experiments $[19,20,23,24,26]$, in comparison with the extremely broad one found in lamellar gradient copolymers.

Consistently with expectations from calorimetric experiments, the comparison between our simulation results for random and lamellar gradient copolymers reveals an extreme dynamic heterogeneity for the second in comparison with the first ones. Relaxation times in the gradient copolymers can differ by many decades depending on the specific position 
along the lamellar domains. This has important consequences for the interpretation of future experimental investigations on the dynamics of these systems. In particular we show that, unlike in lamellar phases of diblock copolymers, in the case of the gradient copolymers the dynamics of the respective homopolymers is not recovered even in the center of the domains, because of the finite probability of finding monomers of the other species at any position. Moreover we find that the dynamics in the interfacial region cannot be modelled by the response of the random copolymer, except in a very narrow region.

The article is organized as follows. In Section II we describe the investigated models and give simulation details. In Sections III and IV we characterize and compare static and dynamic properties of the random and gradient copolymers. We discuss dynamic heterogeneity in the gradient copolymers in terms of position-dependent dynamic correlators. Conclusions are given in Section V.

\section{MODEL AND SIMULATION DETAILS}

\section{Composition profile}

The simulated copolymers contain monomers of two different species $\alpha, \beta$ (see specific notations below) along the chain backbone. Different composition profiles can be achieved in real gradient copolymers by following different synthesis routes. In our work we have assumed the functional form (see e.g., Ref. [54])

$$
p_{\alpha}(i)=\frac{1}{2}\left\{1-\tanh \left[C_{0} \frac{\pi}{N}\left(i-N_{\alpha}\right)\right]\right\}
$$

for the probability distribution of $\alpha$-monomers along the backbone, and $p_{\beta}(i)=1-p_{\alpha}(i)$ for the counterpart $\beta$-monomers. The index $1 \leq i \leq N$ denotes the position of the monomer in the backbone and the parameter $C_{0}$ tunes the shape of the gradient profile. We have fixed a concentration for the majority species $N_{\alpha} / N \approx 0.6$ (see below) and investigated the case $C_{0}=1.8$ for both the bead-spring system and the polyester resin. Lower values of $C_{0}$ lead to a weaker segregation of the two species (see Fig. 1). In order to estimate the effects of the degree of segregation on the dynamics we have performed some additional simulations for the bead-spring system with $C_{0}=1.2$. Having said this, unless otherwise specified, data presented in the following will correspond to the case $C_{0}=1.8$. In the case of the random copolymers the probability distributions $p_{\alpha, \beta}(i)$ are constant, i.e., independent of 
the specific position in the backbone. They are just identical to the respective macroscopic concentrations of $\alpha$ and $\beta$-monomers.

Once the density profiles have been defined, it must be noted that in real random and gradient copolymers each chain has a different specific sequence of $\alpha$ - and $\beta$-monomers. The quantity $p_{\alpha}(i)$ represents the fraction of chains for which the $i$ th-monomer belongs to the $\alpha$-species [55].

\section{Bead-spring model}

We have performed MD simulations of a system of $N_{\text {cha }}=240$ bead-spring chains [37]. We denote the two species as F ('fast') and S ('slow'). Each chain consists of $N=130$ beads, with $N_{\mathrm{F}}=80$ beads of the F-species and $N_{\mathrm{S}}=50$ beads of the S-species. The non-bonded interaction between any two given monomers of the species $\alpha, \beta \in\{\mathrm{F}, \mathrm{S}\}$ is given by a shifted Lennard-Jones potential,

$$
V_{\alpha \beta}(r)=4 \epsilon_{\alpha \beta}\left[\left(\frac{\sigma_{\alpha \beta}}{r}\right)^{12}-\left(\frac{\sigma_{\alpha \beta}}{r}\right)^{6}+\frac{1}{4}\right],
$$

for $r<r_{\mathrm{c}}$ and $V_{\alpha \beta}(r)=0$ for $r \geq r_{\mathrm{c}}$. By using a value $r_{\mathrm{c}}=2^{1 / 6} \sigma_{\alpha \beta}$ for the cut-off distance, the potential is purely repulsive and has no local minima. Moreover, potential and forces are continuous at $r_{\mathrm{c}}$. In addition to the Lennard-Jones potential, two connected beads in a same chain interact through a finitely extensible nonlinear elastic potential (FENE) [37]:

$$
V_{\alpha \beta}^{\mathrm{FENE}}(r)=-\epsilon_{\alpha \beta} K_{\mathrm{F}} R_{0}^{2} \ln \left[1-\left(\frac{r}{R_{0} \sigma_{\alpha \beta}}\right)^{2}\right],
$$

with $K_{\mathrm{F}}=15$ and $R_{0}=1.5$. The sum of the potentials (2) and (3) yields an effective potential between connected monomers that shows a sharp minimum at $r=0.96 \sigma_{\alpha \beta}$ and guarantees chain uncrossability [37].

We have used the same energy scale for the self-interaction, $\epsilon_{\alpha \alpha}=\epsilon=1$, and imposed a strong penalty for the cross-interaction, $\epsilon_{\mathrm{FS}}=9$. With this value the random copolymer forms a microsegregated disordered phase, whereas the gradient copolymer forms a stable ordered lamellar phase (see below). For simplicity we use identical values for the monomer masses, $m_{\mathrm{F}}=m_{\mathrm{S}}=m=1$. We have checked that no significant dynamic asymmetry is obtained by using different values, except for the unrealistic case that $m_{\mathrm{F}}$ and $m_{\mathrm{S}}$ differ by more than a factor of about 10. In analogy with previous simulations of bead-spring polymer 
blends $[56,57]$ and comb copolymers [58], we have introduced size disparity to induce strong dynamic asymmetry. Thus we have used $\sigma_{\mathrm{FF}}=\sigma=1, \sigma_{\mathrm{SS}}=1.6$ and $\sigma_{\mathrm{FS}}=1.3$ for the bead diameters. These values lead to much faster intrinsic dynamics for the F-component than for the S-component (see below).

In the following, temperature $T$, pressure $P$, monomer number density $\rho$, time $t$, and distance will be given respectively in units of $\epsilon / k_{B}$ (with $k_{B}$ the Boltzmann constant), $\epsilon \sigma^{-3}$, $\sigma^{-3}, \sigma(m / \epsilon)^{1 / 2}$, and $\sigma$. Simulation units can be qualitatively mapped to real units as about 5-10 $\AA$ for distances and about 1-10 ps for times (see the discussion in, e.g., Refs. [37, 59]).

Because of their isotropic character, the random copolymer systems were simulated in cubic boxes. Simulation of the lamellar phase of the gradient copolymer requires a more elaborate procedure. Except for the case of very short chains and temperatures far above $T_{\mathrm{g}}$, the spontaneous formation at melt densities — starting from a disordered configuration - of mesophases with well-defined order is unfeasible in simulation time scales. This is a consequence of the extremely long characteristic time scales for chain diffusion [60-63]. Thus, we follow the standard procedure prior to equilibration, and initially construct the system in the expected lamellar geometry [33, 64-66]. Both in the bead-spring and polyester systems we have constructed simulation boxes with six domains (three for each species, see below). We have followed the same procedure for construction and equilibration used by some of us in simulations of lamellar diblock copolymers [33]. Thus, equilibration is performed by combining standard NPT dynamics in the Nosé-Hoover scheme with periodic anisotropic rescaling of the box [64], until the three cartesian components of the internal pressure match the imposed external pressure $(P=2.5)$. See Ref. [33] for details. After this, a further equilibration run is performed at constant density with velocity scaling according to the desired temperature. Production runs are performed in the microcanonical ensemble. The equations of motion are integrated in the Martyna's scheme [67] for the NPT runs and in the velocity-Verlet scheme [68] for the other runs. We use time steps $0.005 \geq \Delta t \geq 0.002$ according to the investigated temperature, which covers the range $0.14 \leq T \leq 0.35$.

The effective packing fraction is defined as $\Phi=(\pi / 6 V) N_{\text {cha }}\left(N_{F} \sigma_{\mathrm{FF}}^{3}+N_{S} \sigma_{\mathrm{SS}}^{3}\right)$, where $V$ is the volume of the simulation box. For the used pressure $P=2.5$ the typical values are $\Phi \approx 0.52$ at all the investigated temperatures, which are characteristic packing fractions for similar bead-spring polymer melts $[37,59]$. For comparison between the dynamics of both components in the copolymer and in the corresponding homopolymer, we haved performed 
additional simulations of the F- and S-homopolymers at $P=2.5$, with chains of $N=70$ and 50 beads respectively.

\section{Polyester resin model}

We have simulated a coarse-grained model of a polyester resin [38] that finds application in the coating industry. The basic chemical units are two dicarboxylic acids and a diol. The acids are adipic acid $\left(\left(\mathrm{CH}_{2}\right)_{4}(\mathrm{COOH})_{2}\right)$ and phthalic acid $\left(\mathrm{C}_{6} \mathrm{H}_{4}(\mathrm{COOH})_{2}\right)$, which in the following will be denoted as $\mathrm{A}$ and $\mathrm{P}$, respectively. The diol is neopentyl glycol $\left(\mathrm{HOCH}_{2}\left(\mathrm{CH}_{3}\right)_{2} \mathrm{CH}_{2} \mathrm{OH}\right)$ and will be denoted as N. Esterification of the acids with the diol units leads to a sequence of dimers, AN and PN, along the chain backbone. Such dimers are the two 'species' that we will consider in the analysis of the structure and dynamics. Accordingly, the gradient copolymer chains have been constructed so that the sequence of ANand PN-units along the backbone obeys the probability distribution of Eq. (1). A random sequence obeying the macroscopic composition is used for the random copolymers.

Coarse-graining of the polyester structure is made with the MARTINI scheme proposed in Ref. [38]. In this scheme, chemical subunits of a few atoms within the AN- and PNunits are coarse-grained to single beads. Namely, each AN- and PN-unit contains four and six beads respectively. The beads interact through coarse-grained potentials, which include non-bonded, bonding and bending interactions. Details about the specific coarse-graining scheme and the parameters of the MARTINI force field can be found in Ref. [38]. The model has been shown to reproduce satisfactorily the properties of the polyester resin in a range of temperatures extending from room temperature up to about $500 \mathrm{~K}$. In this system, the fast component is the one containing adipic acid, AN.

The simulated box contains 160 and 300 polyester chains for the random and gradient copolymer systems, respectively. On average (see footnote [55]) each chain contains 18 ANunits and 12 PN-units (with a total of 144 MARTINI beads for chains with that exact composition). A cubic box is used for the random copolymer, whereas the lamellar phase of the gradient copolymer is prepared by following the same procedure as in the bead-spring system (see above). The system is equilibrated in the NPT ensemble by coupling to the Nosé-Hoover thermostat and Parrinello-Rahman barostat $[69,70]$, with applied pressure $P=1$ bar. Production runs are also performed in the NPT ensemble. We use time steps of 
$20 \mathrm{fs} \geq \Delta t \geq 10 \mathrm{fs}$ according to the investigated temperature, which covers the range $270 \mathrm{~K}$ $\leq T \leq 460 \mathrm{~K}$. For simplicity length units for the polyester will be frequently omitted, giving only numerical values, in particular when comparing with data of the bead-spring system. Unless otherwise specified, distances in the polyester are given in nanometers.

All the simulations of the polyester resin have been performed by using the GROMACS 4.2.2 MD package [71, 72]. A home-made code have been used for the bead-spring simulations. In the next sections we discuss static and dynamic correlators for both models. Static correlators are averaged over 100 equispaced configurations, whereas dynamic correlators are averaged over 20 equispaced time origins. Correlators for the $\mathrm{AN}$ - and PN-units in the polyester must be understood as averaged over all the subunits contained in the former. These subunits will be the MARTINI beads, except in the case of the bond orientational correlators (see below), for which averages are performed over bonds connecting MARTINI beads.

\section{STATIC PROPERTIES}

\section{A. Random copolymers}

Fig. 2 shows two representative snapshots of the two investigated random copolymers. Local microsegregation of the two species can be observed. This finding is consistent with previous observations [49-53]. Segregation is more pronounced for the polyester resin. This can be understood from the fact that the uncompatible AN- and PN-units contain several chemical subunits (beads), in contrast to the simple 'monoatomic' F- and S-units of the beadspring system. Thus, contact between the two species in the polyester is more unfavourable than in the bead-spring system, which results in a stronger segregation for the polyester.

Information about characteristic length scales can be obtained by computing the partial static structure factors,

$$
S_{\alpha \beta}(q)=\frac{1}{\sqrt{N_{\alpha} N_{\beta}}}\left\langle\sum_{i=1}^{N_{\alpha}} \sum_{j=1}^{N_{\beta}} e^{i \mathbf{q} \cdot\left[\mathbf{r}_{i}(0)-\mathbf{r}_{j}(0)\right]}\right\rangle
$$

where $\alpha, \beta \in\{\mathrm{F}, \mathrm{S}\}$ for the bead-spring system and $\alpha, \beta \in\{\mathrm{AN}, \mathrm{PN}\}$ for the polyester system. $N_{\alpha}$ is total number of $\alpha$-monomers. The subscripts $\alpha \beta$ indicate different pair correlations between the chemical species. The brackets denote average over configurations, 
as well as over orientations [73] of the wavevector q. Fig. 3 shows typical results for the partial static structure factors of the bead-spring model. Fig. 4 shows the corresponding results for the polyester resin. All data sets show a sharp first peak. This peak is positive for correlations between chemically identical monomers. On the contrary, a strong anticorrelation (negative peak) is found for chemically distinct monomers. These features reflect the microsegregation of the two species displayed in the snapshots of Fig. 2. The much stronger intensity of the peaks in the polyester system is consistent with the higher degree of segregation observed in the snapshot.

The characteristic size of the microdomains can be estimated from the position of the first peak. The latter is at $q \approx \sigma^{-1}$ and $q \approx 1.3 \mathrm{~nm}^{-1}$, which corresponds to a characteristic size of $d=2 \pi / q \approx 6 \sigma$ and $\approx 5 \mathrm{~nm}$ for the bead-spring and polyester systems, respectively. The second peaks, at $4.5 \sigma^{-1} \lesssim q \lesssim 7 \sigma^{-1}$ and $11 \mathrm{~nm}^{-1} \lesssim q \lesssim 14 \mathrm{~nm}^{-1}$, reflect correlationss at nearest-neighbor distances. Indeed they are located at $q \approx 2 \pi / \sigma_{\alpha \beta}$, with $\sigma_{\alpha \beta}$ the length scale of the non-bonded $\alpha$ - $\beta$ interaction. Finally, the oscillations at higher- $q$ values just correspond to higher-order harmonics. The domain size is esentially temperature-independent, as illustrated in Fig. 5. Indeed the position of the peak is not significantly affected by varying $T$. The growing of the intensity as temperature decreases just reflects the usual localization arising from the lower mobility of the particles.

\section{B. Gradient copolymers}

Fig. 6 shows typical snapshots of the bead-spring and polyester gradient copolymers. Both systems form an ordered lamellar phase that remains stable at all the simulated temperatures. No drifts in the thermodynamic, structural and dynamic observables have been found in the simulation time scale. The lamellar structure consists of domains rich in one species and poor in the other. Unlike for the case of strongly segregated lamellar phases of diblock copolymers, where both species only coexist in the interfacial region, monomers of one species can be found even in the center of a domain rich in the other species. This feature can be quantified by computing the normalized local density along the direction $z$ perpendicular to the lamellar plane. For the bead-spring model we compute the quantity $\rho_{\alpha}^{*}(z)=n_{\alpha}(z) \sigma_{\alpha \alpha}^{3} /\left(L_{x} L_{y} \delta\right)$, where $L_{x}$ and $L_{y}$ are the box sides in the directions parallel to the lamellar planes, and $n_{\alpha}(z)$ is the number of $\alpha$-monomers within the slab $(z, z+\delta)$. The 
factor $\sigma_{\alpha \alpha}^{3}$ is included for a proper comparison between the density profiles of both compo-

nents. In the case of the polyester we compute the mass density, $\rho_{\alpha}^{(\mathrm{m})}(z)=n_{\alpha}(z) m_{\alpha} /\left(L_{x} L_{y} \delta\right)$, with $m_{\alpha}$ the mass of the $\alpha$-unit. We use a slab thickness of $\delta=0.5 \sigma$ and $0.1 \mathrm{~nm}$ for the bead-spring and polyester system, respectively. Fig. 7 shows representative results for the density profiles of both systems. The data reflect the existence of broad interfacial regions separating the lamellar domains, and a finite probability of finding monomers of a given species at any position, even in the center of the domains rich in the other species.

\section{DYNAMIC PROPERTIES}

\section{A. Random copolymers}

Fig. 8 shows the temperature dependence of the mean squared displacement (MSD), $\left\langle\Delta r_{\alpha}^{2}(t)\right\rangle$, of the $\alpha$-monomers in the bead-spring $(\alpha \in\{\mathrm{F}, \mathrm{S}\})$ and polyester $(\alpha \in\{\mathrm{AN}, \mathrm{PN}\})$ systems. In the case of the bead-spring system we have included some results for the respective homopolymers. It can be seen that, as a result of linking to a component of different intrinsic mobility, the dynamics of the slow $(\mathrm{S})$ and fast $(\mathrm{F})$ monomers in the random copolymer is respectively sped up and slowed down in comparison to the homopolymer. After the initial ballistic regime, $\left\langle\Delta r_{\alpha}^{2}(t)\right\rangle \sim t^{2}$, the MSD shows, for sufficiently low temperatures, a plateau. The plateau extends over longer time windows as temperature decreases. This behavior reflects the characteristic caging regime exhibited by the monomers on approaching the glass transition $[59,74,75]$. However, the caging effect is much more pronounced (stronger localization and longer duration) for the S- and PN-monomers than for the F-and AN-monomers respectively. This dynamic asymmetry is apparently more pronounced in the simulated bead-spring model than in the polyester. In both systems the dynamic asymmetry increases by decreasing the temperature. Having noted this, because of the chemical links between both species, the dynamic asymmetry is restricted to a limited length scale. Thus, after decaging (departure from the plateau) the MSD of both species merge for displacements of just a few bead diameters $\left(\left\langle\Delta r^{2}(t)\right\rangle \sim 10\right)$. This feature resembles observations in self-assembled comb copolymers [58, 76-79], for which backbones and side groups microsegregate in disordered domains, in a similar fashion as the random copolymers investigated here. On the other hand, the former feature is rather different from the scenario found 
in dynamically asymmetric polymer blends. In such systems the absence of links between both components allows for a full decoupling of their relaxation time scales (even by many decades) and, at low temperatures, the fast component can perform large-scale diffusion even at time scales for which the slow matrix has not completed its structural relaxation [80-82].

Fig. 9 shows results for the $T$-dependence of the bond orientational correlator, $P_{\alpha}(t)=$ $\langle\cos (\theta(t))\rangle$, for the $\alpha$ - $\alpha$ bonds, where $\theta(t)$ is the angle between the bond vector at time $t=0$ and at the considered $t$. The results in Fig. 9 show similar trends to those found in the MSD. Again the effects are more pronounced in the bead-spring model than in the polyester. Interestingly, the caging effect in the reorientational behavior is stronger for the AN-component than for the PN-one (see plateaux for $P(t)>0.8$ in Fig. 9). This behavior is rather different from that observed in the bead-spring system, and even in the MSD of the same polyester system. However, the expected trend - faster relaxation of the AN-AN bonds - is recovered at longer times; see the crossing of the solid and dashed lines for both $T=350$ and $390 \mathrm{~K}$. Presumably, this crossing will also occur for $T<350 \mathrm{~K}$, though the respective time scales are beyond the limit of the simulation window.

\section{B. Gradient copolymers}

Figs. 10 and 11 show results for the MSD and bond correlator in the gradient copolymer. As observed in the random copolymer, the system exhibits dynamic asymmetry. Again, the latter becomes more pronounced as temperature decreases. On passing, we note that in both the random and gradient copolymers, the caging regime is not followed by the transition to the long-time diffusive regime observed in simple glass formers. Instead, we find apparent subdifussive behavior up to the limit of the simulation window. To illustrate this effect we have included in Figs. 10 and 11 dashed-dotted lines representing power-law behavior, $\left\langle\Delta r_{\alpha}^{2}(t)\right\rangle \sim t^{x<1}$, for comparison with the simulation data. It is well-known that, as a consequence of chain connectivity, simple polymers exhibit subdiffusive Rouse-like behavior $(x=1 / 2)$ after the segmental dynamics [83]. Even further subdiffusive regimes are observed prior to diffusion in the case of long entangled polymers. The latter are usually explained in terms of reptational dynamics $[83,84]$. Though characterization of chain dynamics is beyond the scope of this work, it is worth commenting that the presence of dynamic asymmetry 
might alter significantly the Rouse regime commonly observed in homopolymers. Several works on dynamically asymmetric blends have revealed, for the fast component, strong deviations from Rouse dynamics below the entanglement length of the homopolymer and down to the segmental scale [56, 85-87]. Some of us have proposed [87] that the usual Rouse assumption [83] of uncorrelation of the external forces, acting on the (fast) tagged chain, is not valid when the latter is surrounded by a much slower matrix (the slow component in the blend). Accordingly, memory effects, introduced through a generalized Langevin equation [88], may account for the observed deviations from the Rouse model. Whether a related or even more complex scenario can be found for the chain dynamics of the copolymers investigated here - showing compositional heterogeneity at the intramolecular and even at the large-scale structural level - is an open question for future investigations.

Coming back to the local dynamics, the data in the gradient copolymer show important qualitative differences with results for the random copolymer. Thus, no merging of the MSD of both components is found within the simulation time scale. Note that whereas in the random copolymer merging occurs for values of $\left\langle\Delta r_{\alpha}^{2}(t)\right\rangle \sim 10$, the gradient copolymer still exhibits a clear separation of time scales at such values of the MSD. More qualitative differences are revealed by analyzing the van Hove self-correlation functions, $G_{\mathrm{s}}(r ; t)$, at the former time scales. The insets of Fig. 12 show results for the fast component (F- and AN-monomers). The van Hove functions have been multiplied by the phase factor $4 \pi r^{2}$. With this correction, the data in the insets represent the histogram of displacements at the selected times. Results for the gradient and random copolymers are shown at temperatures and times (see caption) for which the respective MSD's have the same value. Moreover, the selected cases correspond to situations far beyond the caging regime in the MSD. Indeed the latter takes values $\left\langle\Delta r_{\alpha}^{2}(t)\right\rangle \sim 4$, corresponding to average displacements of about two monomer diameters. Despite having the same value of $\left\langle\Delta r_{\alpha}^{2}(t)\right\rangle$, the selected cases exhibit very different features in the van Hove functions. Whereas a nearly Gaussian form is found for the random copolymer, the histogram for the gradient copolymer exhibits a complex structure (see insets of Fig. 12). This shows a well-defined peak at short distances $r \approx 0.5$, i.e, smaller than the monomer size. The peak is followed by a broad shoulder extending up to distances of $r>5$. Thus, the van Hove function reveals that there is a significant group of monomers that remain trapped in the cage, whereas many others have moved several times their own size. The latter are even more than in the random copolymer with the same MSD 
(compare the tails of the respective histograms in the insets of Fig. 12).

The stronger non-Gaussian character of the relaxation in the gradient copolymer can also be demonstrated by computing the non-Gaussian parameter, $\alpha_{2}(t)=3\left\langle\Delta r^{4}(t)\right\rangle /\left(5\left\langle r^{2}(t)\right\rangle^{2}\right)$. This parameter quantifies deviations from Gaussianity in the van Hove function $\left(\alpha_{2}(t)=0\right.$ for a strictly Gaussian function). Results of $\alpha_{2}(t)$ for both species in the bead-spring models are shown in Figs. 13 and 14, for the random and gradient copolymer respectively. The results for the random copolymer are qualitatively similar to those found in simple glassformers. As time increases $\alpha_{2}(t)$ grows up from zero to a maximum. This maximum takes place at the time scale of the decaging in the MSD (compare big squares in Figs. 8a and 13). At longer times, $\alpha_{2}(t)$ progressively decreases up to the limit $\alpha_{2}(t \rightarrow \infty)=0$ expected for Gaussian diffusion. However, the non-Gaussian parameter for the gradient copolymer exhibits a much more complex behavior (see Fig. 14). For the fast F-monomers a maximum is found at the time scale of the decaging (compare big squares in Figs. 10a and 14a). However, after this maximum $\alpha_{2}(t)$ does not show the monotonic decay to zero found for the random copolymer. Instead, it forms a minimum and shows a new increase that persists during the whole simulation time window, even reaching values higher than the maximum at the decaging time (see data for $T>0.15$ in Fig. 14a). There is an increasing non-Gaussianity even at times for which the average displacement is of several times the monomer size (compare, e.g., data at $T=0.17$ and $t \sim 10^{5}$ for the F-momoners in Figs. 10a and 14a). In the case of the S-monomers, the behaviour of $\alpha_{2}(t)$ is even more complex. It does not form a well-defined maximum at the time scale of the decaging in the MSD (compare big circles in Figs. 10a and 14b). As much, an apparent plateau - note the poor statistics - is formed in the $\alpha_{2}(t)$ of the S-monomers prior to the further increase at longer times.

The complex, highly non-Gaussian, behavior of the van Hove function in the gradient copolymer suggests that the dynamics in this phase is extremely heterogeneous, even at time scales far beyond the caging regime of the MSD. Now we show that this feature is a direct consequence of the gradients of composition which are present in the lamellar structure of the gradient copolymer. Monomers of a given species can be surrounded mostly by monomers of the same or the other species according to the domain (rich in one or the other species) in which they are located. They can also be located in the broad interfacial region, in which both species coexist in relevant concentrations. These different environments can lead to very different mobilities of the monomers in the lamellar phase. In order to characterize 
the dynamic heterogeneity, we compute van Hove functions as a function of the positions of the monomers in the domains. First, we define the slab $\Delta z_{\min }<\Delta z<\Delta z_{\max }$ where $\Delta z$ is the distance to the nearest interfacial plane. The latter is defined at the intersection between the corresponding density profiles of the two species (see Fig. 7). Then we compute dynamic correlators for the monomers that, at the time origin $(t=0)$, are located in the former slab. We use small values for the slab thickness, $\Delta z_{\max }-\Delta z_{\min }=\sigma$ and $1 \mathrm{~nm}$ for the bead-spring and polyester system, respectively. These are sufficiently small to solve fine details of the position-dependence of the dynamics, and at the same time provide good statistics for a broad range of $\Delta z$-values. In the following, we denote the distance $\Delta z$ to the nearest interfacial plane with positive or negative signus if the monomers are located in the domain rich or poor in their species, respectively. For each slab we compute the function $G_{\mathrm{s}}(\Delta z, r ; t)$, which measures the probability of the monomers initially located in the slab to move a radial distance $r$ at time $t$. The corresponding histograms of displacements, for F- and AN-monomers, are shown in the main panels of Fig. 12, together with the global functions $4 \pi r^{2} G_{\mathrm{S}}(r ; t)$ discussed above. The results presented for the different distances $\Delta z$ reveal a dramatic dependence of the monomer displacements on their initial positions. For the temperatures of Fig. 12, the F- and AN-monomers initially located around the interfacial plane, $-1 \lesssim \Delta z \lesssim 1$, remain trapped there at the end of the simulation. This is also true for those fast monomers located in the center of the slow (S- and PN-rich) domains, corresponding to data for $\Delta z \ll 0$ (not shown). On the contrary, fast monomers initially located within the fast (F-and AN-rich) domains become increasingly more mobile at longer distances $(\Delta z \gg 0)$ from the interfacial plane. These results provide a strong connection between the local composition in the gradient copolymer and the mobility of the monomers. Those being surrounded preferently by F- or AN-monomers become highly mobile. Likewise, monomers surrounded preferently by S- or PN-monomers remain localized within the cage $r \lesssim 1$ formed by these.

Following a procedure analogous to that described above, we compute the slab-dependent MSD, $\left\langle\Delta r^{2}(\Delta z ; t)\right\rangle$. Results for the two components of both the bead-spring and polyester system are shown in Figs. 15 and 16 at selected temperatures. We remind that, for slabdependent correlators of the species $\alpha$, values $\Delta z>0$ correspond to slabs in the domain rich in $\alpha$-monomers, whereas values $\Delta z<0$ correspond to slabs in the $\alpha$-poor domain. The results in Figs. 15 and 16 provide a direct comparison between the dynamics at the 
different slabs over the whole time window. As can be seen, a strong dynamic heterogeneity arises already at the beginning of the caging regime. The plateau height, which reflects the strength of the localization during the caging regime $[74,75]$, is strongly dependent on the position along the lamellar structure. Fast (F- and AN-) monomers in the interfacial region or in the slow (S- and PN-rich) domains show a much stronger localization than those in the center of the fast (F-and AN-rich) domains. Likewise, decaging for the former ones occurs at much longer times. Results for the slow (S- and PN-) monomers show the opposite trends. Slow monomers become much more mobile in the center of the fast domains than in the interface or in the center of the slow domains. By estimating slab-dependent $\alpha$-relaxation times as those for which $\left\langle\Delta r^{2}(\Delta z ; t)\right\rangle \sim \sigma_{\alpha \alpha}^{2}$, it can be seen that these can differ by time decades according to the specific value of $\Delta z$. This feature can also be observed in the slab-dependent bond correlators, $P(\Delta z ; t)$. Fig. 16 shows representative results for the F-F and AN-AN bonds. Thus, whereas for the selected $T=270 \mathrm{~K}$ relaxation of the AN-AN bonds in the AN-rich domain is nearly completed at $3 \mathrm{~nm}$ from the interfacial plane, the corresponding bonds at the same distance, but located in the PN-rich domain, only start to relax at the end of the simulation window. Similar trens (see Supp. Info.) are found by computing the former observables as a function of the monomer position $1 \leq i \leq N$ along the chain contour. Of course, the reason for this analogy is that in lamellar structures $i$ and $\Delta z$ are strongly correlated quantities.

Results in Figs. 15 and 16 show that, for fixed temperature, relaxation times are extremely dependent on the distance to the interfacial plane. Moreover, inspection of the slab-dependent van-Hove functions reveals that, not only the time scale, but also the nature of the relaxation can be very different along the domains. To illustrate this, we show in Fig. 17 the former functions at $T=0.14$ for the F-monomers in two selected slabs. At long distances from the interfacial plane (slab $7<\Delta z<8$, see panel (a)) the histogram of displacements shows a smooth shape at all times, with the exception of a shoulder at $t \sim 10^{3}$ arising at distances $r \gtrsim 0.5$, which suggests some heterogeneity in the decaging process of the particles at the former slab. A more complex behavior is found at shorter distances from the interface (slab $3<\Delta z<4$, see panel (b)). A double-peak structure is observed at intermediate times. As time increases, the first peak in the histogram decreases, whereas the second one increases. The positions of the two peaks are nearly constant $\left(r_{1} \approx 0.3\right.$ and $\left.r_{2} \approx 0.9\right)$ up to time scales of $t \sim 10^{5}$. According to these observations, particles are 
trapped within a well-defined distance and escape by performing jumps with a well-defined amplitude. This suggests a strong hopping-like character for the relaxation at slabs close to the interface, and resembles observations in complex disordered mixtures with components of very different mobilities and, more generally, in crowded environments [89]. The 'discrete' nature of the motion persists even at times for which half of the particles have moved beyond their own size $(r>1)$ - see data in Fig. $17 \mathrm{~b}$ for $t=10^{5}$, where the first peak is still clearly visible. Only at much longer times the double-peak structure vanishes and Gaussian behavior is recovered in the van Hove function.

In Figs. 15 and 16 we have included the global MSD's and bond correlators (i.e., averaged over all monomers and bonds of the respective species), for comparison with the slab-dependent quantities. This comparison illustrates how the global response - the one usually measured in experiments - arises from a extremely broad distribution of relaxation times in the lamellar structure of the gradient copolymer. In Figs. 15a and 16a we also include the MSD and bond correlator of the F-homopolymer at the same temperature. It can be seen that the homopolymer dynamics is not recovered at any position of the lamellar phase. Even in the center of the F-rich domains $(\Delta z \sim 8)$, there is a strong difference between the relaxation of the F-species in the gradient copolymer and in the F-homopolymer. This feature is very different from the observation in strongly segregated lamellae of diblock copolymers, in which the dynamics of the homopolymer is recovered at the center of the domain $[33,34]$. The origin of this difference with diblock copolymers is the finite probability of finding monomers of both species at every position in the lamellar gradient copolymer, which modifies the original relaxation of the homopolymers.

As mentioned in Section II, the degree of segregation in the gradient copolymer can be tuned by changing the value of $C_{0}$ in eq. (1). Namely, larger values of $C_{0}$ lead to a stronger segregation. All the results presented above correspond to the case $C_{0}=1.8$. We have performed some additional simulations at $T=0.14$ for the bead-spring model with $C_{0}=1.2$, corresponding to a weaker degree of segregation (see Fig. 1). In Fig. 18 we show results, at $T=0.14$, for the slab-dependent MSD and bond correlators of the F-component in both cases $C_{0}=1.2$ and 1.8. For comparison we also include the corresponding results for the F-homopolymer. It can be seen that relaxation of the F-component is systematically faster, for all values of $\Delta z$, in the case $C_{0}=1.8$ than in the case $C_{0}=1.2$. The same trend is found in the global relaxation, probed by $\left\langle\Delta r^{2}(t)\right\rangle$ and $P(t)$ (not shown), which is consistent 
with dielectric spectroscopy experiments on gradient copolymers with different degrees of segregation [26]. Since the system $C_{0}=1.8$ is more strongly segregated, for fixed $\Delta z$ the fast F-monomers are, on average, surrounded by less slow S-monomers, which facilitates their mobility. The opposite trend is found for the S-monomers (not shown). In the system $C_{0}=1.8$ they are surrounded by less fast F-monomers and therefore they exhibit slower relaxation than in the system $C_{0}=1.2$. On the other hand, since in the system $C_{0}=1.8$ the monomers have a lower probability of finding monomers of the other species in their surroundings, relaxation in the center of the domains is closer to that of the respective homopolymer (filled squares in Fig. 18).

Finally, we discuss the effect of temperature on the observed dynamic heterogeneity. We have estimated slab-dependent relaxation times $\tau(\Delta z)$ as those for which the slab-dependent bond correlator takes the value $P(\Delta z ; \tau)=e^{-1}$. Fig. 19b shows the temperature dependence of these relaxation times for the gradient copolymer. For comparison we include results for the F- and S-component in the random copolymer, as well as for the F-homopolymer. The latter are again obtained by using $P(\tau)=e^{-1}$, where $P(t)$ is the global correlator for the respective component, and are shown in both panels of Fig. 19. Panel (a) shows such data for global relaxation over a broader temperature range than in panel (b), and includes the corresponding results for the F-component in the gradient copolymer. Consistently with previous findings by lattice simulations [16] and dielectric spectroscopy [26], the global segmental relaxation in the gradient copolymer is, at fixed temperature, intermediate between that in the homopolymer and in the random copolymer.

No data have been shown in Fig. 19 for the global response of the S-component in the gradient copolymer, since in the investigated $T$-range for the gradient copolymer the former is extremely slow and does not relax within the simulation time window. Concerning the dynamic response of the different slabs, we observed that indeed most of the S-S bonds in the gradient copolymer do not relax in the investigated $T$-range. Relaxation times at such temperatures could only be measured for the S-S bonds located beyond the interfacial plane $(\Delta z<0)$, in the F-rich domain. Moreover we have not included, even if some of them do relax, data for bonds with $\Delta z<-4$, i.e, data for bonds initially located in the domain rich in the other species and relatively far from the interfacial plane. The reason is that, because of the low population of such bonds, the respective correlators have poor statistics and it is difficult to obtain reliable relaxation times. 
The representation of Fig. 19b reveals important features. Thus, the dispersion in relaxation times along the lamellar domains becomes stronger as temperature decreases. Actually, the dispersion is even stronger than that shown in the figure, because of long relaxation times beyond the simulation window that have not been included (see above). This observation is consistent with the experimental finding of an extremely broad glass transition in lamellar gradient copolymers $[10,17,19,20,23,24,26,35]$, in contrast to the narrow transition observed in random copolymers $[19,20,23,24,26]$.

A tempting picture for describing the experimental global response in lamellar gradient copolymers might be considering the latter as a superposition of three contributions: two contributions as those of the respective homopolymers and a third one as that of the random copolymer. The former two might be expected to originate from relaxation in the center of the domains, whereas the third one would arise in the interface. Data in Fig. 19 shows that this approximation is incorrect. The dynamics of the homopolymer is approached only in the limit of high $T$ and long distances $(\Delta z>8)$ from the interfacial plane. For low $T$, homopolymer dynamics is not recovered at any distance, and deviations become more pronounced as temperature decreases. On the other hand, relaxation in the interfacial region is extremely sensitive to position, and the dynamics of the random copolymer is only found in a very narrow region of the gradient copolymer $(0<\Delta z<1$ for the F-component) [90].

\section{CONCLUSIONS}

By means of computer simulations, we have performed a detailed investigation of structural and dynamic properties in disordered random copolymers and in lamellar phases of gradient copolymers. The copolymers contain two kinds of monomers with very different mobilities. Two models have been investigated: i) a generic bead-spring model and ii) a coarse-grained model of a polyester resin, by using the MARTINI force field. We have found that the qualitative features found for the generic bead-spring system are reproduced in the realistic model of the polyester. This suggests that such features will be generally found in real random and gradient copolymers.

The lamellar phase of the simulated gradient copolymers is formed by domains rich in one of the two chemical species and poor in the other one. The domains are separated by broad interfaces, and because of the gradient-like composition of the chains, monomers of both 
species have significant concentrations at any position of the domains. As a consequence, monomers can probe rather different chemical environments in the lamellar gradient copolymer. Because of this strong compositional heterogeneity and the strong dynamic asymmetry between the two species, monomers of a given species can be located both in highly mobile and in quasi-arrested environments. Hence, relaxation is characterized by an extreme dynamic heterogeneity, even at time scales far beyond the caging regime of the global dynamic response. This is confirmed by an analysis of dynamic correlators as a function of the distance to the interfacial plane. In the case of random copolymers long-range ordering is not possible, and local microsegregation results in a much weaker dynamic heterogeneity. The former features are consistent with the experimental observation of narrow glass transitions in random copolymers but extremely broad ones in lamellar gradient copolymers.

Our results have important consequences for the interpretation of future experimental investigations on the dynamics of lamellar gradient copolymers. Unlike in lamellar phases of diblock copolymers, in the case of the gradient copolymers the dynamics of the respective homopolymers is not recovered even in the center of the domains, because of the finite probability of finding monomers of the other species at any position. Moreover, the dynamics in the interfacial region cannot be modelled by the response of the random copolymer, except in a very narrow region.

\section{ACKNOWLEDGEMENTS}

Part of this work was carried out under the HPC-Europa2 project (project number: 228398) with the support of the European Commission Capacities Area-Research Infrastructures Initiative. This project granted us CPU time at GENCI-CINES (Paris, France). We also acknowledge support from the projects MAT2007-63681 (Spain), MAT2012-31088 (Spain) and IT-436-07 (GV, Spain). We thank Luca Monticelli for useful discussions. M. Z. S. acknowledges the colleagues of INSERM UMR-S 665 for their kind reception.

[1] Bates, F. S.; Fredrickson, G. H. Annu. Rev. Phys. Chem. 1990, 41, 525.

[2] Matsen, M. W.; Bates, F. S. Macromolecules 1996, 29, 1091.

[3] Hamley, I. W. The Physics of Block Copolymers; Oxford University Press: Oxford, UK, 1998. 
[4] Hadjichristidis, N.; Pispas, S.; Floudas, G. Block Copolymers: Synthetic Strategies, Physical Properties, and Applications; Wiley-IEEE: Hoboken, NJ, 2003.

[5] Kryszewski, M. Polym. Adv. Technol. 1998, 9, 244.

[6] Matyjaszewski, K.; Ziegler, M. J.; Arehart, S. V.; Greszta, D.; Pakula, T. J. Phys. Org. Chem. 2000, 13,775 .

[7] Davis, K. A.; Matyjaszewski, K. Adv. Poly. Sci. 2002, 159, 1.

[8] Beginn, U. Colloid Polym. Sci. 2008, 286, 1465.

[9] Zaremski, M. Y.; Kalugin, D. I.; Golubev, V. B. Polym. Sci. A 2009, 51, 103.

[10] Mok, M. M.; Kim, J.; Wong, C. L. H.; Marrou, S. R.; Woo, D. J.; Dettmer, C. M.; Nguyen, S. T.; Ellison, C. J.; Shull, K. R.; Torkelson, J. M. Macromolecules 2009, 42, 7863.

[11] Aksimentiev, A.; Holyst, R. J. Chem. Phys. 1999, 111, 2329.

[12] Lefebvre, M. D.; Olvera de la Cruz, M.; Shull, K. R. Macromolecules 2004, 37, 1118.

[13] Jian, R.; Jin, Q.; Li, B.; Ding, D.; Wickham, R. A.; Shi, A. C. Macromolecules 2008, 41, 5457.

[14] Tito, N. B.; Milner, S. T.; Lipson, J. E. G. Macromolecules 2010, 43, 10612.

[15] Ganesan, V.; Kumar, N. A.; Pryamitsyn, V. Macromolecules 2012, 45, 6281.

[16] Pakula, T.; Matyjaszewski, K. Macromol. Theory Simul. 1996, 5, 987.

[17] Gray, M. K.; Zhou, H.; Nguyen, S. T.; Torkelson, J. M. Macromolecules 2004, 37, 5586.

[18] Lefebvre, M. D.; Dettmer, C. M.; McSwain, R. L.; Xu, C.; Davila, J. R.; Composto, R. J.; Nguyen, S. T.; Shull, K. R. Macromolecules 2005, 38, 10494.

[19] Kim, J.; Mok, M. M.; Sandoval, R. W.; Woo, D. J.; Torkelson, J. M. Macromolecules 2006, $39,6152$.

[20] Wong, C. L.; Kim, J.; Torkelson, J. M. J. Polym. Sci. B 2007, 45, 2842.

[21] Jouenne, S.; González-León, J. A.; Ruzette, A. V.; Lodefier, P.; Tencé-Girault, S.; Leibler, L. Macromolecules 2007, 40, 2432.

[22] Mok, M. M.; Pujari, S.; Burghardt, W. R.; Dettmer, C. M.; Nguyen, S. T.; Ellison, C. J.; Torkelson, J. M. Macromolecules 2008, 41, 5818.

[23] Mok, M. M.; Kim, J.; Torkelson, J. M. J. Polym. Sci. B 2008, 46, 48.

[24] Jakubowski, W.; Juhari, A.; Best, A.; Koynov, K.; Pakula, T.; Matyjaszewski, K. Polymer 2008, 49, 1567.

[25] Singh, N., Tureau, M. S.; Epps, T. H. Soft Matter 2009, 5, 4757. 
[26] Mok, M. M.; Masser, K. A.; Runt, J.; Torkelson, J. M. Macromolecules 2010, 43, 5740.

[27] Mok, M. M.; Ellison, C. J.; Torkelson, J. M. Macromolecules 2011, 44, 6220.

[28] Donth, E. The Glass Transition; Springer: Berlin-Heidelberg, Germany, 2001.

[29] Kyritsis, A.; Pissis, P.; Mai, S. M.; Booth, C. Macromolecules 2000, 33, 4581.

[30] Lorthioir, C.; Alegría, A.; Colmenero, J.; Deloche, B. Macromolecules 2004, 37, 7808.

[31] Lund, R.; Willner, L.; Alegría, A.; Colmenero, J.; Richter, D. Macromolecules 2008, 41, 511.

[32] Sanz, A.; Nogales, A.; Ezquerra, T. A. Soft Matter 2011, 7, 6477.

[33] Slimani, M. Z.; Moreno, A. J., Colmenero, J. Macromolecules 2011, 44, 6952.

[34] Slimani, M. Z.; Moreno, A. J., Colmenero, J. Macromolecules 2012, 45, 8841.

[35] Hashimoto, T.; Tsukahara, Y.; Tachi, K.; Kawai, H. Macromolecules 1983, 16, 648.

[36] Mayes, A. M.; Johnson, R. D.; Russell, T. P.; Smith, S. D.; Satija, S. K.; Majkrzak, C. F. Macromolecules 1993, 26, 1047.

[37] Kremer, K.; Grest, G. S. J. Chem. Phys. 1990, 92, 5057.

[38] Rossi, G.; Giannakopoulos, I.; Monticelli, L.; Rostedet, N. K. J.; Puisto, S. K.; Lowe, C.; Taylor, A. C.; Vattulainen, I.; Ala-Nissila, T. Macromolecules 2011, 44, 6198.

[39] Marrink, S. J.; de Vries, A. H.; Mark, A. E. J. Phys. Chem. B 2004, 108, 750.

[40] Marrink, S. J.; Risselada, H. J.; Yefimov, S.; Tieleman, D. P.; de Vries, A. H. J. Phys. Chem. $B$ 2007, 111, 7812 .

[41] Monticelli, L.; Kandasamy, S. K.; Periole, X.; Larson, R. G.; Tieleman, D. P., Marrink, S. J.; J. Chem. Theory Comput. 2008, 4, 819.

[42] de Jong, D. H.; Singh, G.; Bennett, W. F. D.; Arnarez, C.; Wassenaar, T. A.; Schafer, L. V.; Periole, X.; Tieleman, D. P.; Marrink, S. J.; J. Chem. Theory Comput. 2013, 9, 687.

[43] Lee, H.; de Vries, A. H.; Marrink, S. J.; Pastor, R. W. J. Phys. Chem. B 2009 113, 13186.

[44] Rossi, G.; Monticelli, L.; Puisto, S. R.; Vattulainen, I.; Ala-Nissila, T. Soft Matter 2011, 7, 698.

[45] Lee, H.; Pastor, R. W. J. Phys. Chem. B 2011 115, 7830.

[46] Perlmutter, J. D; Drasler, W. J.; Xie, W.; Gao, J.; Popot, J. L.; Sachs, J. N. Langmuir 2011, 27, 10523.

[47] Milani, A.; Casalegno, M.; Castiglioni, C.; Raos, G. Macromol. Theory Simul. 2011, 20, 305.

[48] Fredrickson, G. H.; Milner, S. T. Phys. Rev. Lett. 1999, 67, 835.

[49] Gee, R. H.; Fried, L. E.; Cook, R. C. Macromolecules 2001, 34, 3050. 
[50] Alsunaidi, A.; Abu-Sharkh, B. F. J. Chem. Phys. 2003, 119, 9894.

[51] Houdayer, J.; Müller, M. Macromolecules 2004, 37, 4283.

[52] Steinmüller, B.; Müller, M.; Hambrecht, K. R.; Smith, G. D.; Bedrov, D. Macromolecules 2012, 45, 1107.

[53] Steinmüller, B.; Müller, M.; Hambrecht, K. R.; Bedrov, D. Macromolecules 2012, 45, 9841.

[54] Zhao, Y.; Luo, Y. W.; Ye, C.; Li, B. G.; Zhu, S. J. Polym. Sci. A 2009, 47, 69.

[55] For each chain we generate the composition pattern as follows. We make a loop over all monomers $1 \leq i \leq N$. For each $i$ we generate a random number $\xi$ and assign it to the species $\alpha$ if $\xi \leq p_{\alpha}(i)$; otherwise we asign it to the species $\beta$. This procedure does not give an identical fraction $N_{\alpha} / N$ for all chains, but introduces some polydispersity in the composition of the chains. In the case of the polyester we have kept this polydispersity (which is closer to the experimental situation). In the case of the bead-spring system, the chemical patterns of the different chains have been 'rebalanced' to fixed composition by exchanging the chemical identity of randomly selected monomers along the chains. This does not alter the average gradient profile, which still obeys Eq. (1). After this procedure, all bead-spring chains have exactly 80 F-monomers and 50 S-monomers.

[56] Moreno, A.J.; Colmenero, J. J. Chem. Phys. 2006, 124, 184906.

[57] Moreno, A.J.; Colmenero, J. Phys. Rev. Lett. 2008, 100, 126001.

[58] Moreno, A.J.; Arbe, A.; Colmenero, J. Macromolecules 2011, 44, 1695.

[59] Baschnagel, J.; Varnik, F. J. Phys.: Condens. Matter 2005, 17, R851.

[60] Hoffmann, A.; Sommer, J. U.; Blumen, A. J. Chem. Phys. 1997, 106, 6709.

[61] Murat, M.; Grest, G. S.; Kremer, K. Macromolecules 1999, 32, 595.

[62] Anderson, J. A.; Lorenz, C. D.; Travesset, A. J. Chem. Phys. 2008, 128, 184906.

[63] Moreno, A. J.; Colmenero, J. Macromolecules 2009, 42, 8543.

[64] Schultz, A. J.; Hall, C. K.; Genzer, J. J. Chem. Phys. 2004, 120, 2049.

[65] Loison, C.; Mareschal, M.; Kremer, K.; Schmid, F. J. Chem. Phys. 2003, 119, 13138.

[66] Grest, G. S.; Lacasse, M. D.; Kremer, K.; Gupta, A. M. J. Chem. Phys. 1996, 105, 10583.

[67] Martyna, G. J.; Tuckerman, M. E.; Tobias, D. J.; Klein, M. L. Mol. Phys. 1996, 87, 1117.

[68] Frenkel, D.; Smit, B. Understanding Molecular Simulation; Academic Press (Elsevier): San Diego, 1996.

[69] Nose, S.; Klein, M. L. Mol. Phys. 1983, 50, 1055. 
[70] Parrinello, M.; Rahman, A. J. Appl. Phys. 1981, 52, 7182.

[71] Berendsen, H. J. C.; van der Spoel, D.; van Drunen, R. Comp. Phys. Comm. 1995, 91, 43.

[72] http://www.gromacs.org

[73] For each modulus $q$ we include all wavevectors compatible with the periodic boundary conditions and, in order to improve statistics, within a small tolerance of $\pm 0.01 q$.

[74] Binder, K.; Kob, W. Glassy Materials and Disordered Solids; World Scientific: Singapore, 2005

[75] Barrat, J. L.; Baschnagel, J.; Lyulin, A. Soft Matter 2010, 6, 3430.

[76] Beiner, M.; Huth, H. Nature Mater. 2003, 2, 595.

[77] Hiller, S.; Pascui, O.; Kabisch, O.; Reichert, D.; Beiner, M. New J. Phys. 2004, 6, 10.

[78] Arbe, A.; Genix, A. C.; Colmenero, J.; Richter, D.; Fouquet, P. Soft Matter 2008, 4, 1792.

[79] Gerstl, C.; Schneider, G. J.; Pyckhout-Hintzen, W.; Allgaier, J.; Willbold, S.; Hofmann, D.; Disko, U.; Frielinghaus, H.; Richter, D. Macromolecules 2011, 44, 6077.

[80] Lutz, T. R.; He, Y. Y.; Ediger, M. D.; Cao, H. H.; Lin, G. X.; Jones, A. A.; Macromolecules 2003, 36, 1724.

[81] Colmenero, J.; Arbe, A. Soft Matter 2007, 3, 1474.

[82] Maranas, J. K. Curr. Opin. Colloid Interface Sci. 2007, 12, 29.

[83] Doi, M.; Edwards, S. F. The Theory of Polymer Dynamics; Oxford University Press: Oxford, U. K., 1986.

[84] McLeish, T. C. B. Adv. Phys. 2002, 51, 1379.

[85] Brodeck, M.; Alvarez, F.; Moreno A. J.; Colmenero, J.; Richter, D. Macromolecules 2010, 43, 3036.

[86] Arrese-Igor, S.; Alegría, A.; Moreno, A. J.; Colmenero, J. Macromolecules 2011 44, 3611.

[87] Arrese-Igor, S.; Alegría, A.; Moreno, A. J.; Colmenero, J. Soft Matter 2012 8, 3739.

[88] Schweizer, K. S.; Fuchs, M.; Szamel, G.; Guenza, M.; Tang, H Macromol. Theory Simul. 1997, 6, 1037.

[89] Special section on Complex dynamics of fluids in disordered and crowded environments, Coslovich, D.; Kahl, G.; Krakoviack, V. (eds). J. Phys.: Condens. Matter 2011, 23, 230302.

[90] One might speculate that the dynamics in each slab is equivalent to that of a random copolymer with the adequate composition (close to symmetric for slabs in the interfacial region and strongly asymmetric for slabs in the centers of the domains). To test this idea, extensive 
simulations of random copolymers would be necessary, at different concentrations and several temperatures for each composition. 


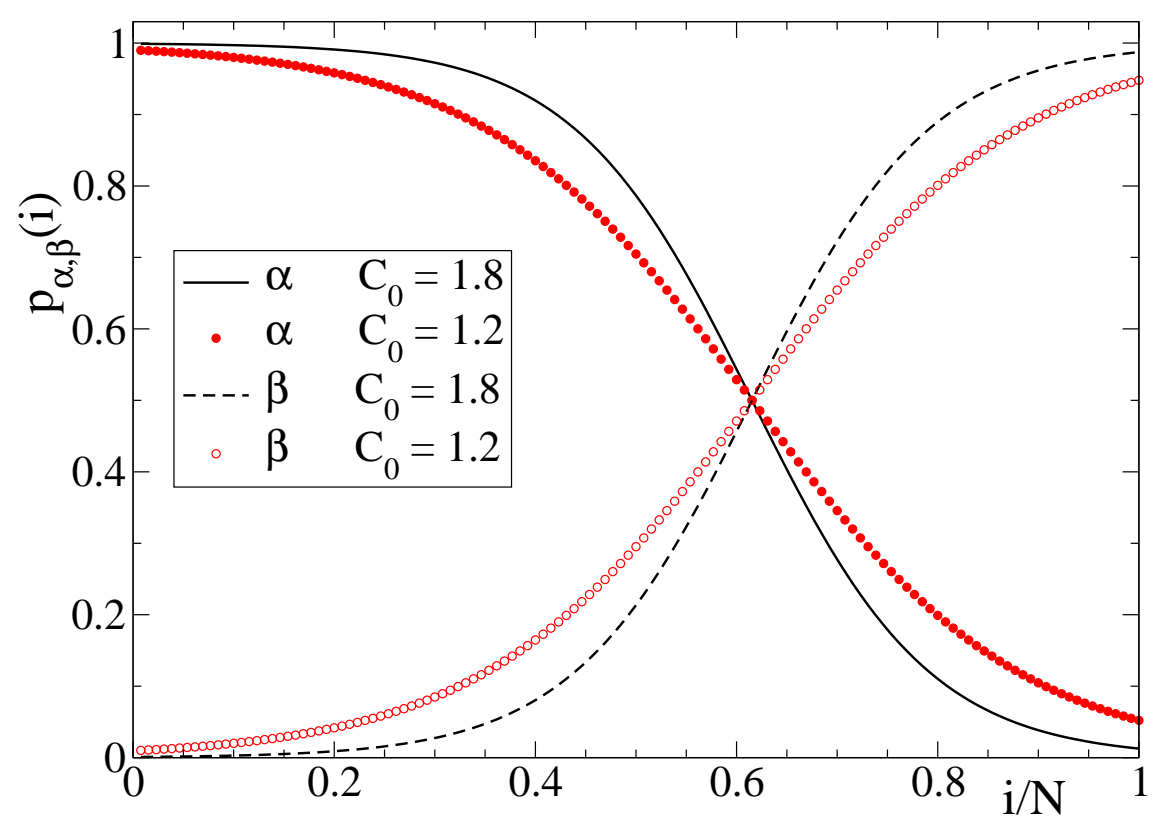

FIG. 1: Probability distribution of $\alpha$ - and $\beta$-monomers along the chain backbone of the gradient copolymer, for composition $N_{\alpha} / N=0.615$, corresponding to the bead-spring model (see below). The shape parameters are $C_{0}=1.8$ (lines) and 1.2 (symbols). 


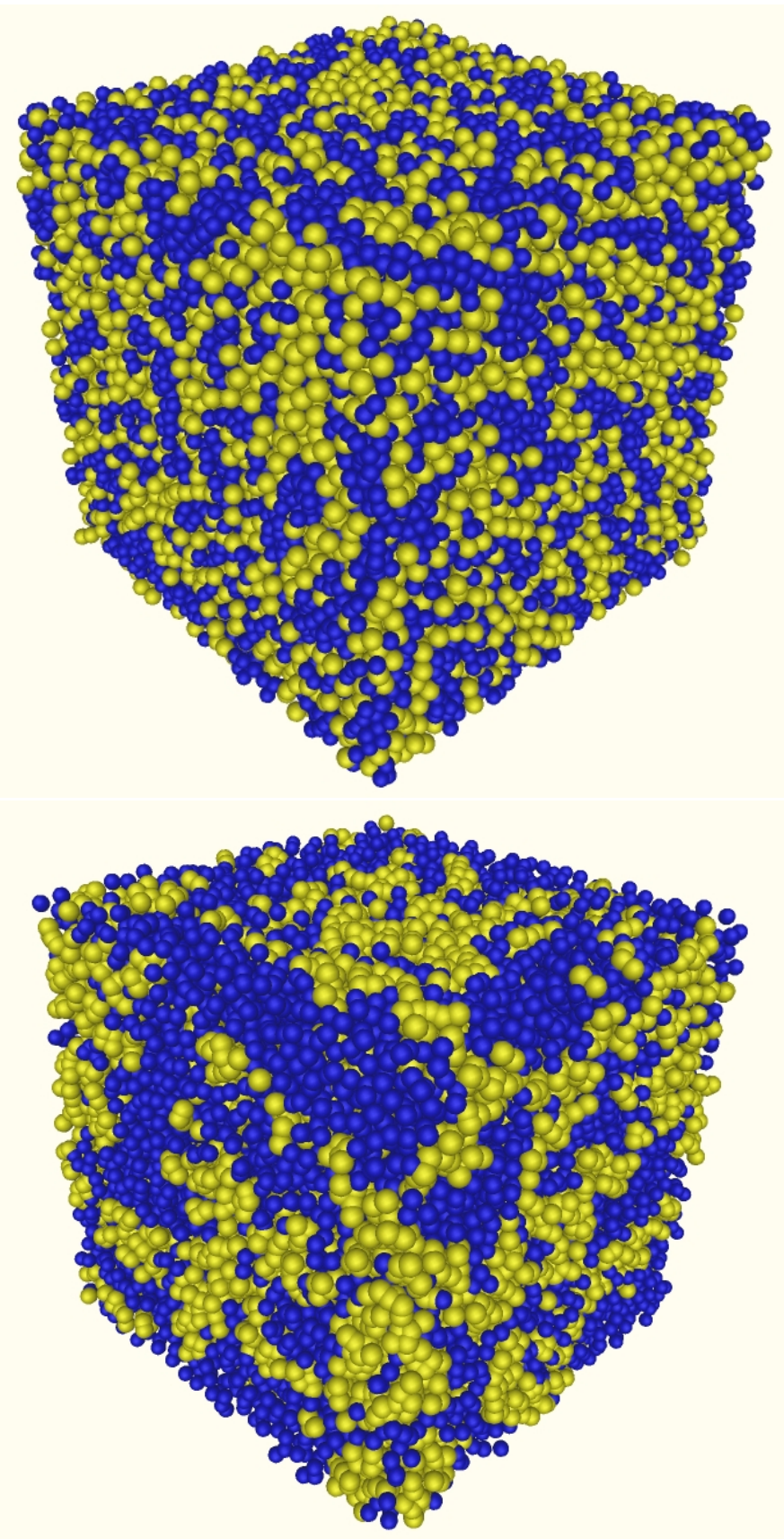

FIG. 2: Typical snapshots of the random copolymer. Top panel: bead-spring system. Bottom panel: polyester resin. F- and AN- monomers are represented as blue beads. S- and PN-monomers are represented as yellow beads. 

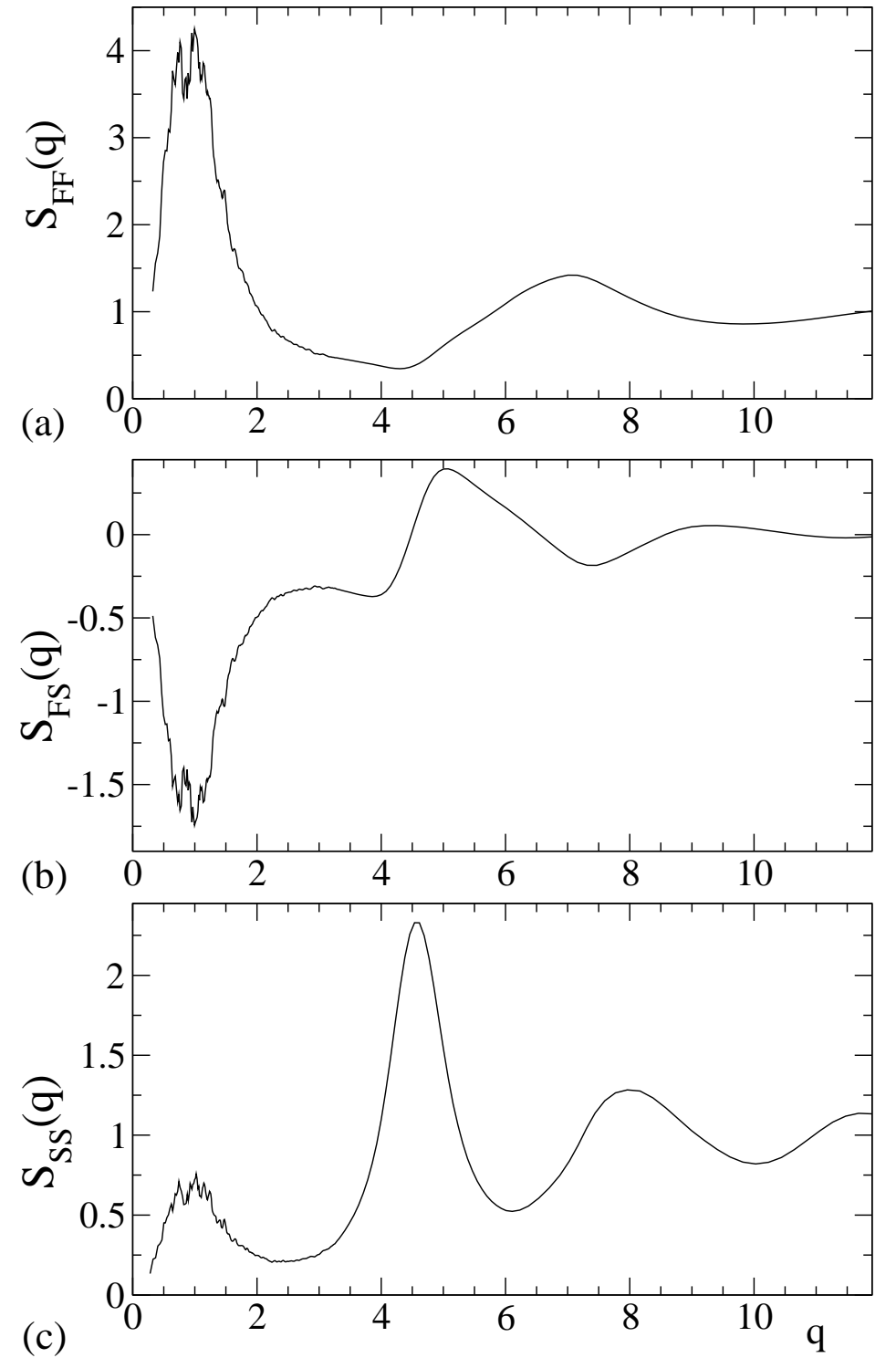

FIG. 3: Partial static structure factors for the bead-spring model at $T=0.25$. 


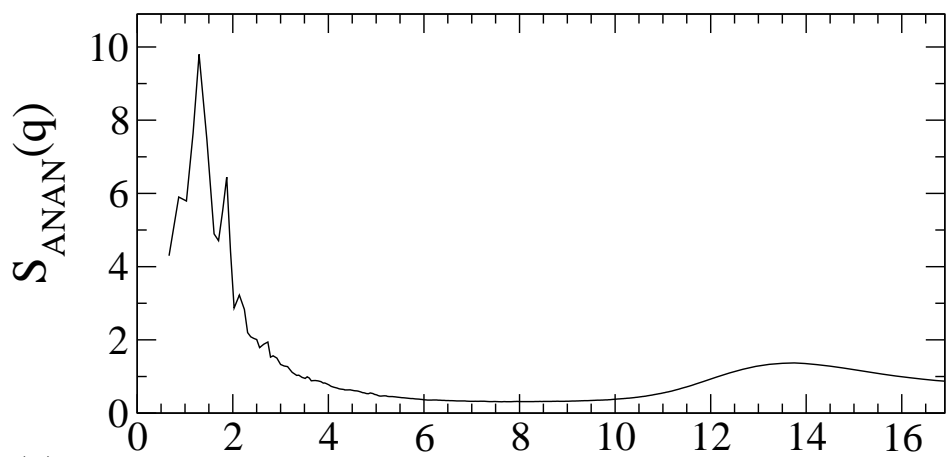

(a)
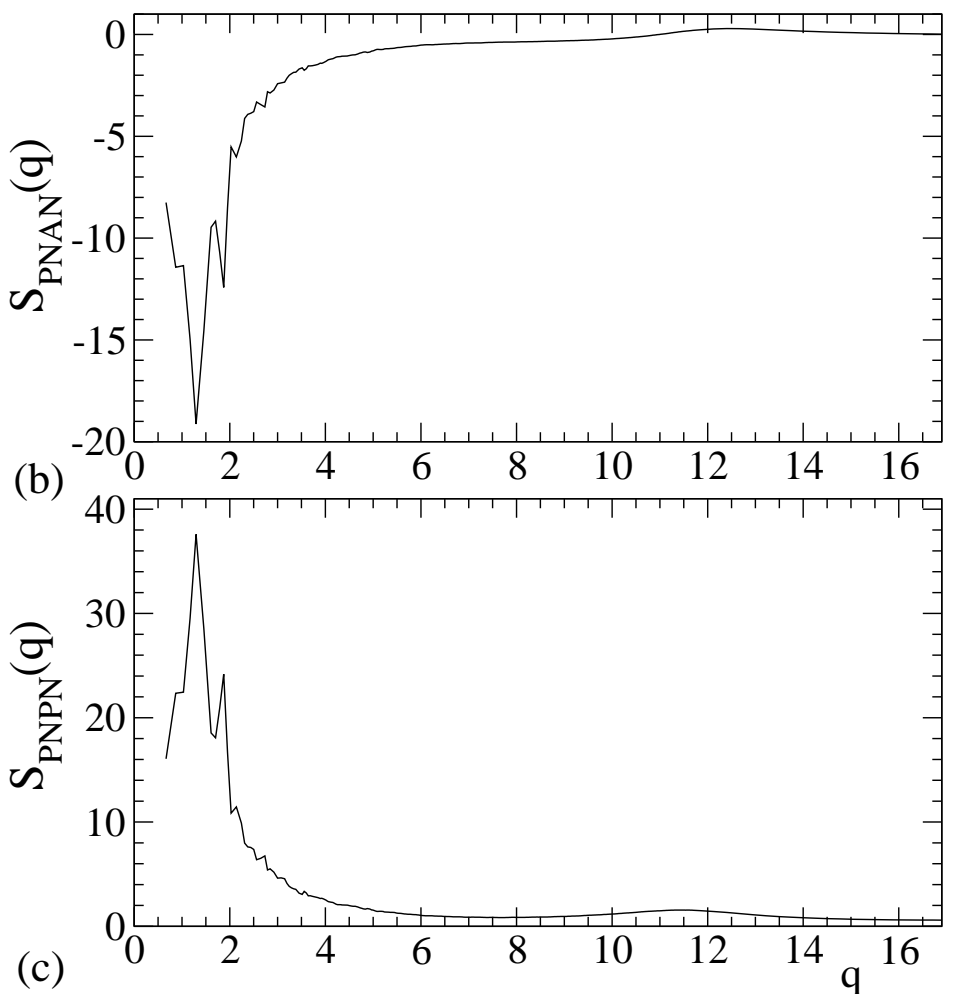

FIG. 4: Partial static structure factors for the polyester resin at $T=350 \mathrm{~K}$. 

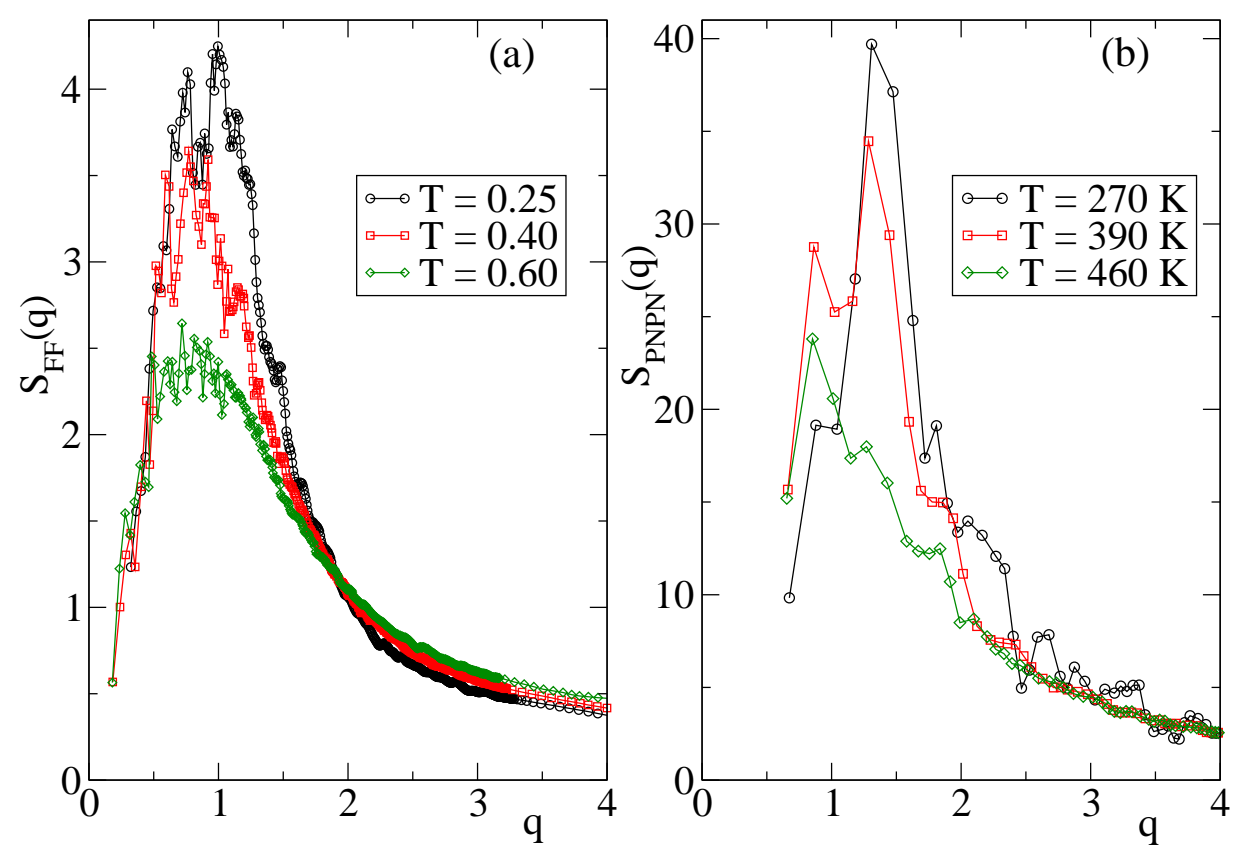

FIG. 5: Temperature dependence of the first peak in the partial static structure factors for F-F (a) and PN-PN pairs (b). 


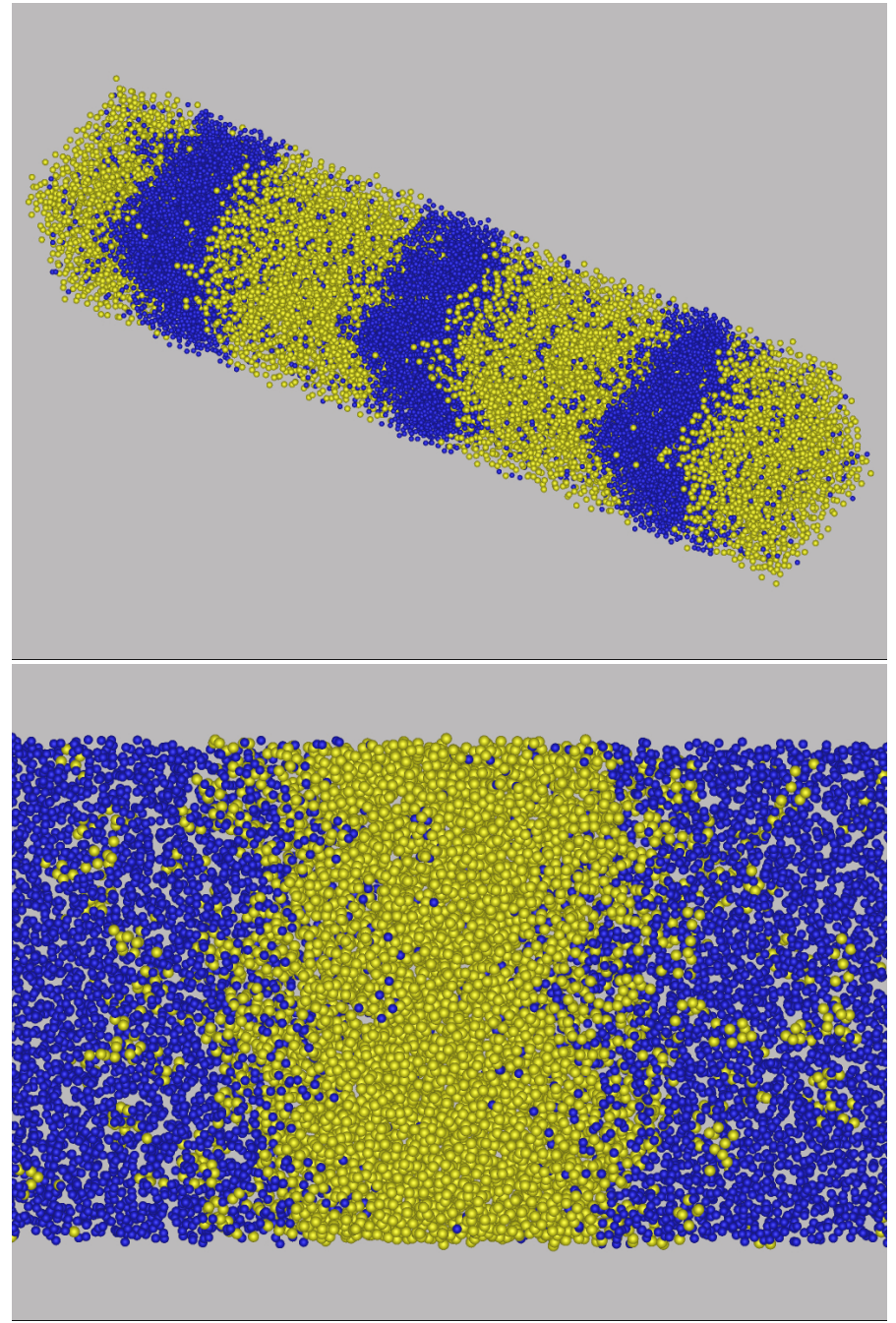

FIG. 6: Typical snapshots for the bead-spring and polyester gradient copolymers (top and bottom panels respectively). Blue beads represent F- and AN-monomers. Yellow beads represent S- an PN-monomers. The bead size has been reduced for the sake of clarity. In both cases the box contains six domains. The bottom panel shows a portion of the box, in order to highlight the gradients of composition along the domains. 

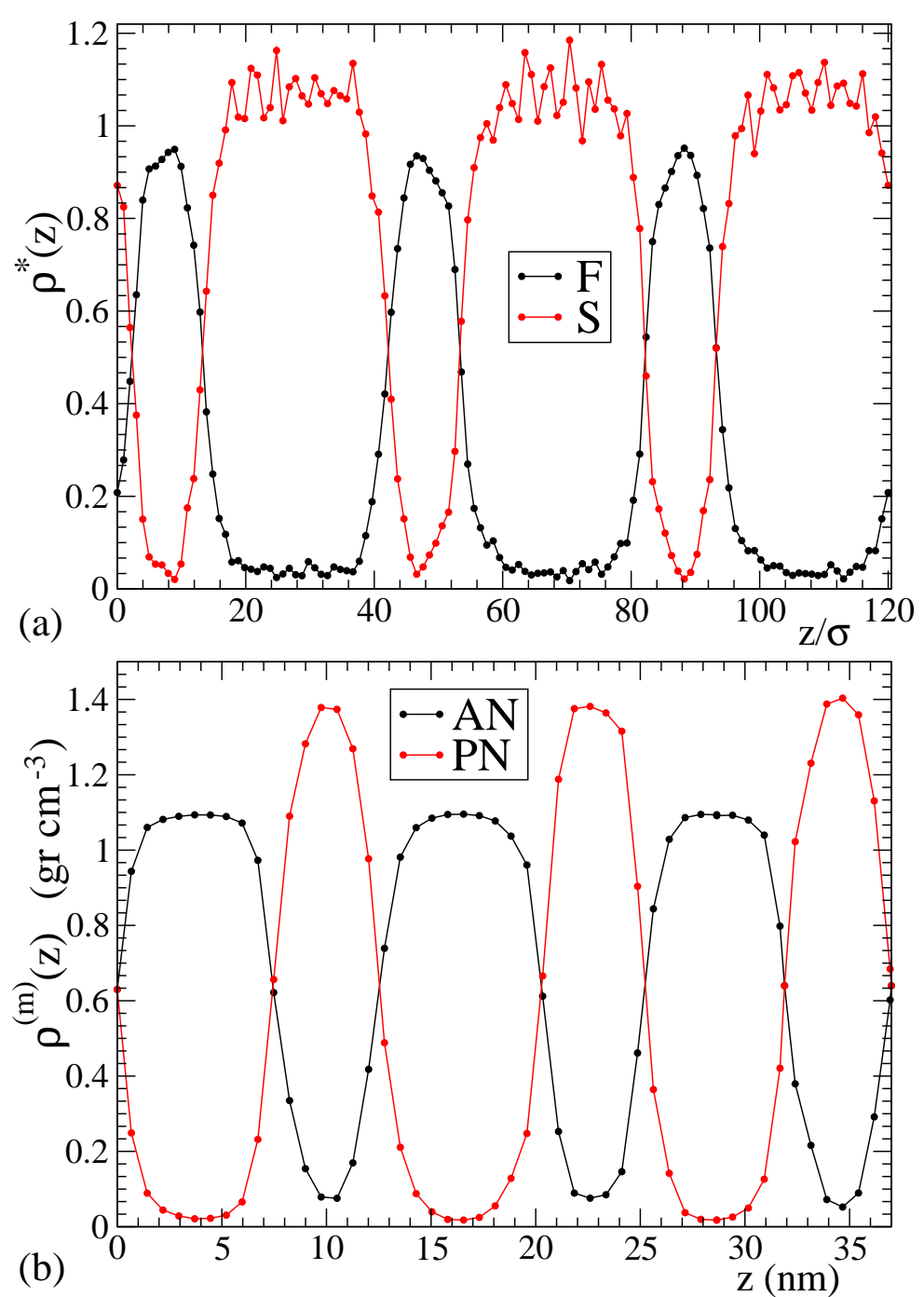

FIG. 7: Density profiles along the direction perpendicular to the lamellar planes. The $z$-values cover the whole box side $L_{z}$. (a): data for the bead-spring model at $T=0.14$. (b): data for the polyester at $T=320 \mathrm{~K}$. 

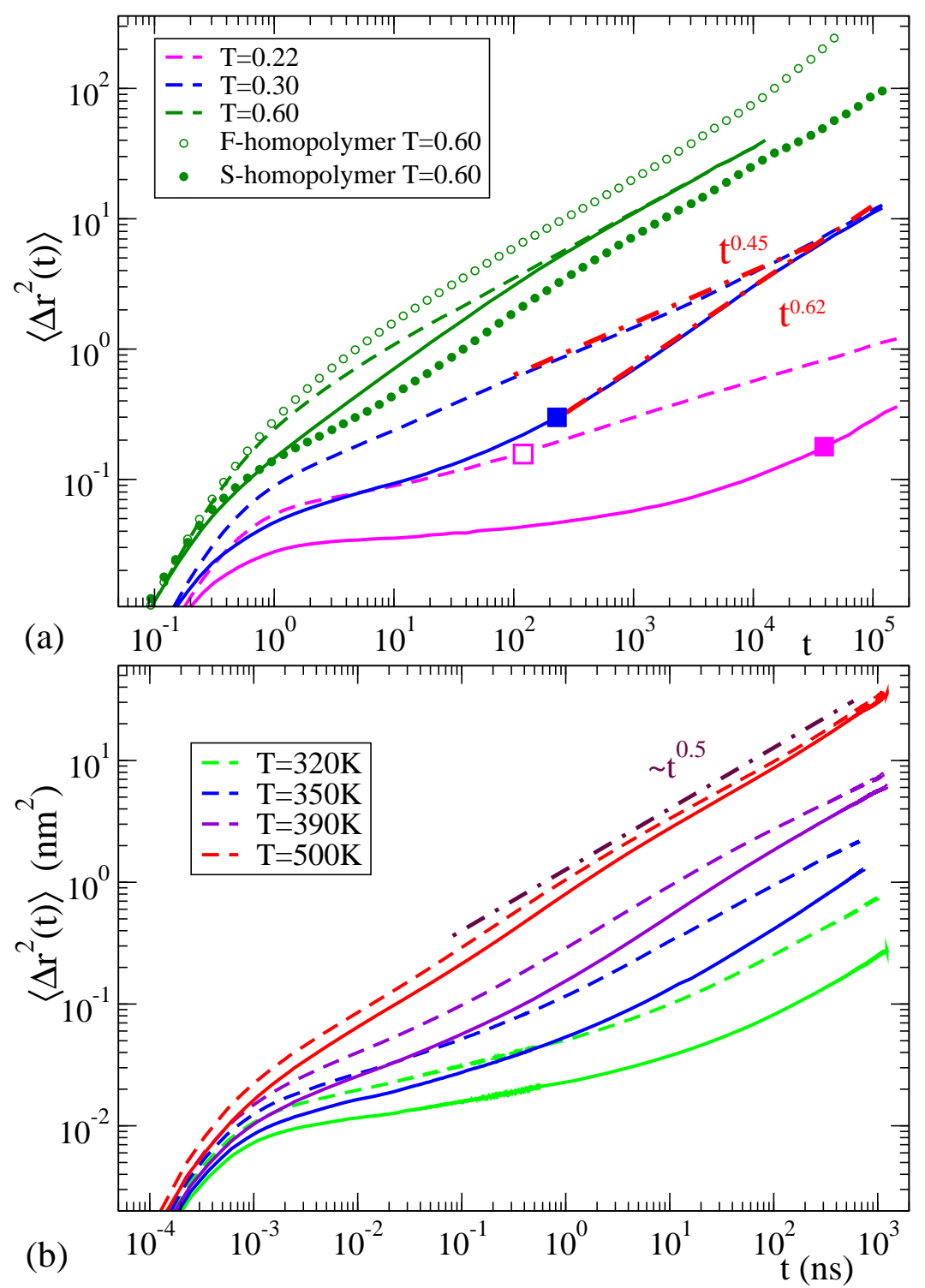

FIG. 8: Temperature dependence of the MSD in the random copolymer, for the bead-spring (a) and polyester (b) systems. Dashed lines correspond to F- and AN-monomers. Solid lines correspond to S- and PN-monomers. Data sets for a same temperature are represented with same colours. Symbols in panel (a) are results, at $T=0.60$, for the F- (small open symbols) and S-homopolymer (small filled symbols). Dashed-dotted lines represent power-law behavior $\sim t^{x}$ (exponents are indicated), and are included for comparison with the simulation data. For some data sets, we have added big squares in panel (a) of this figure and in Fig. 13. Same codes for big squares in both figures correspond to same times, temperatures and monomer species. Specifically, such times correspond to the maxima of the respective non-Gaussian parameters (see Fig. 13). 

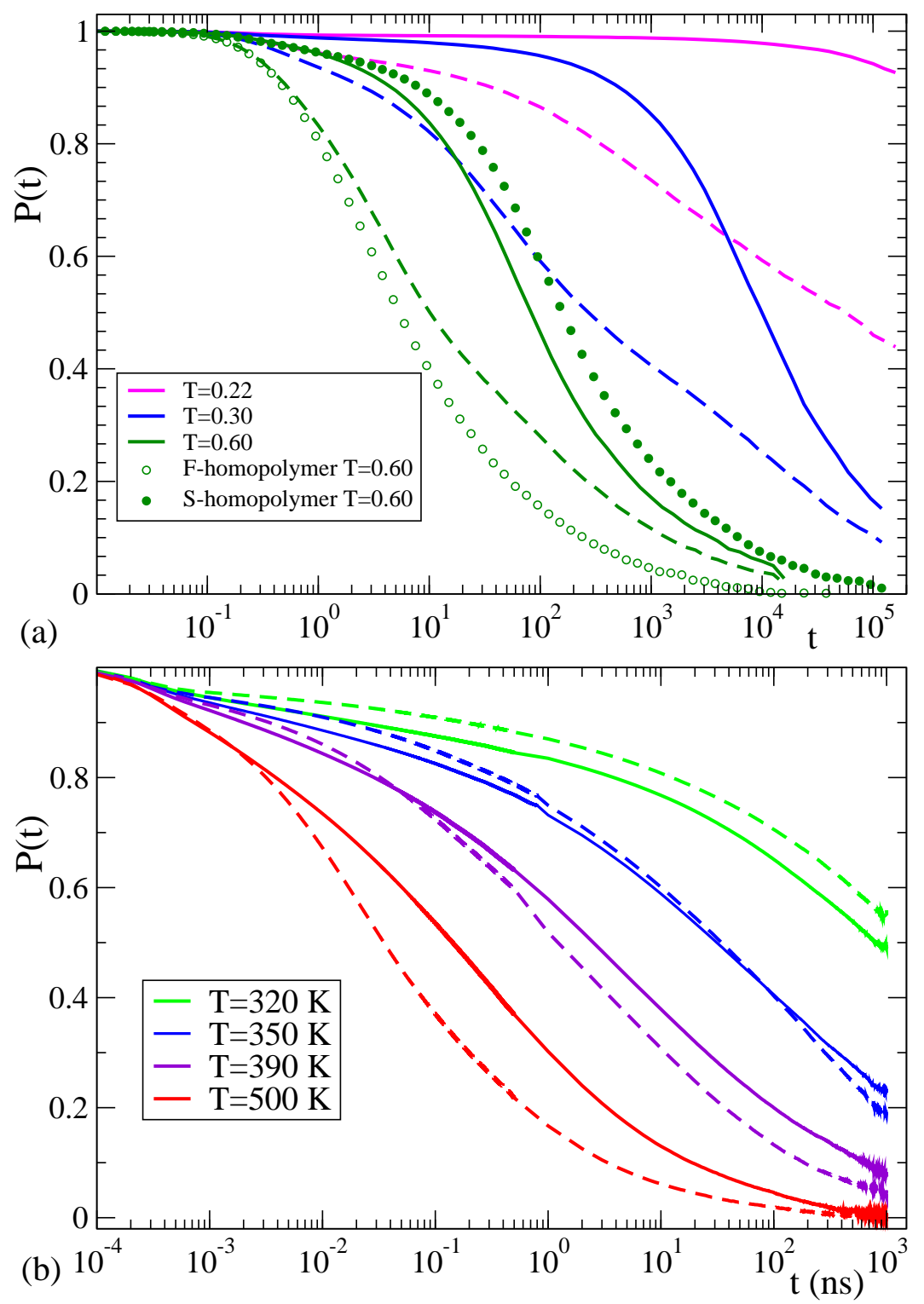

FIG. 9: Temperature dependence of the bond orientational correlator in the random copolymer, for the bead-spring (a) and polyester (b) systems. Dashed lines correspond to F-F and AN-AN bonds. Solid lines correspond to S-S and PN-PN bonds. Data sets for a same temperature are represented with same colours. Symbols in panel (b) are results, at $T=0.60$, for the F- (open symbols) and S-homopolymer (filled symbols). 


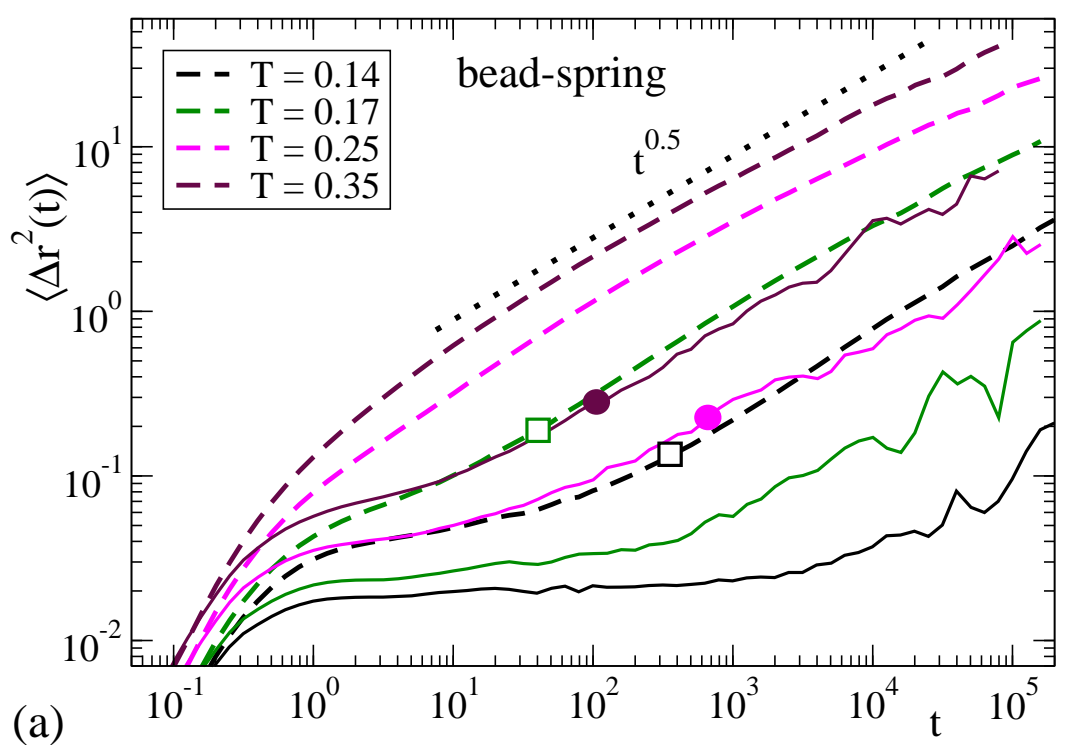

(a)

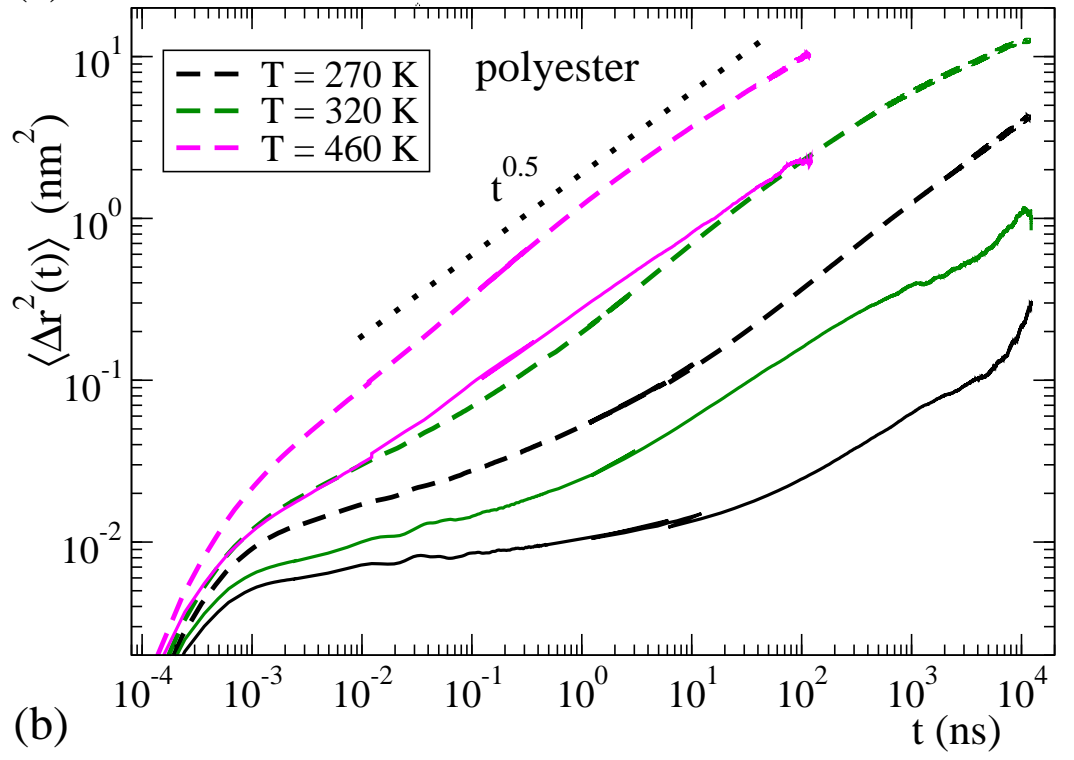

FIG. 10: Temperature dependence of the MSD in the gradient copolymer for the bead-spring (a) and polyester system (b). Dashed lines are data for F- and AN-monomers. Solid lines are data for S- and PN-monomers. Dashed and solid lines for a same temperature are plotted with the same colours (see legend). Dashed-dotted lines represent power-law behavior $\sim t^{0.5}$ and are included for comparison with the simulation data. For some data sets, we have added big symbols in panel (a) of this figure and in Fig. 14. Same codes for big symbols in both figures correspond to same times, temperatures and monomer species (see Fig. 14). 


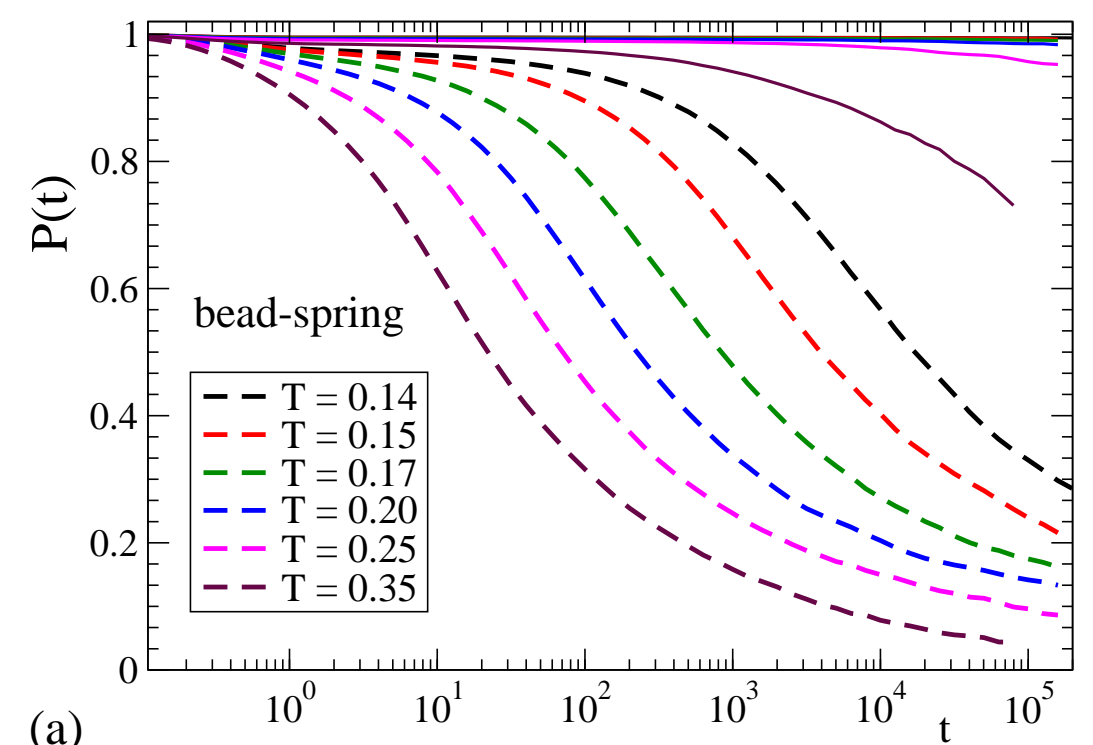

(a)

(b)

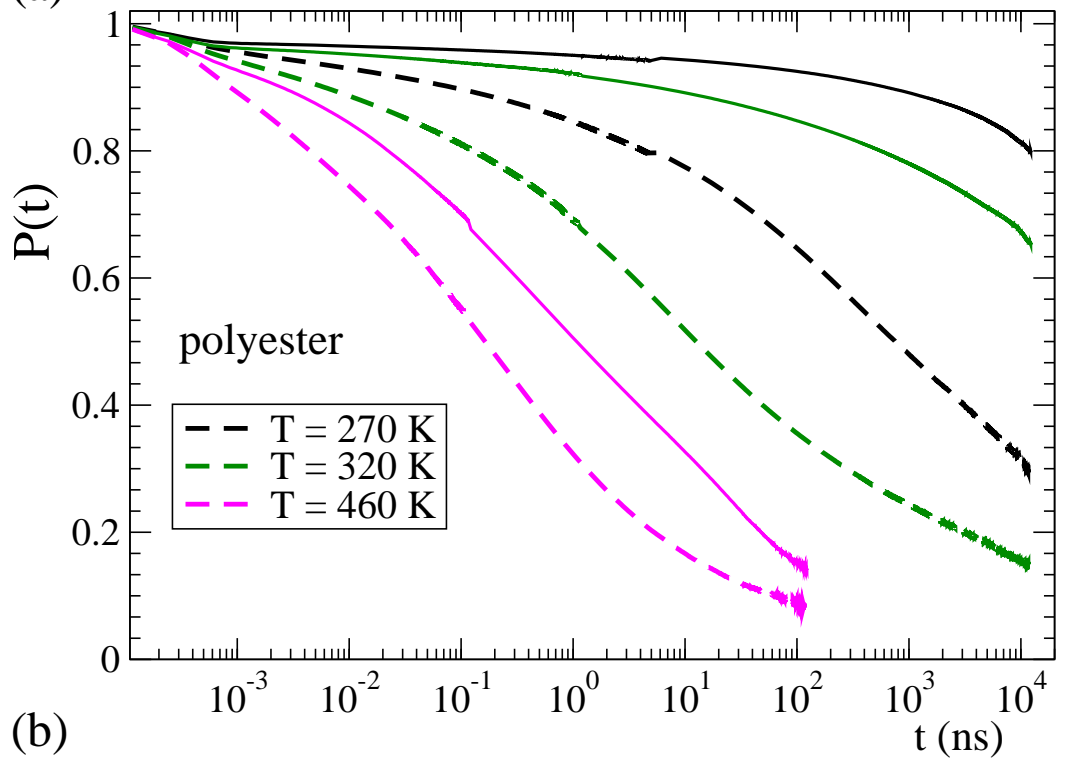

FIG. 11: Temperature dependence of the bond orientational correlator in the gradient copolymer for the bead-spring (a) and polyester system (b). Dashed lines are data for F-F and AN-AN bonds. Solid lines are data for S-S and PN-PN bonds. Dashed and solid lines for a same temperature are plotted with the same colours (see legend). 


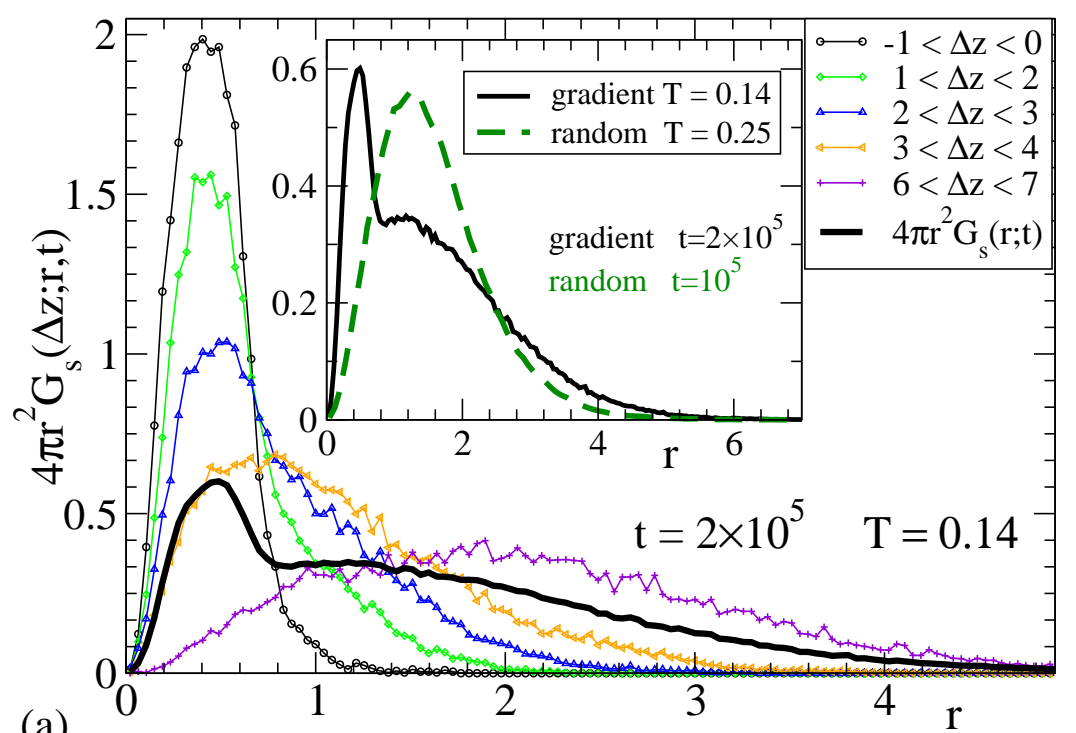

(a)

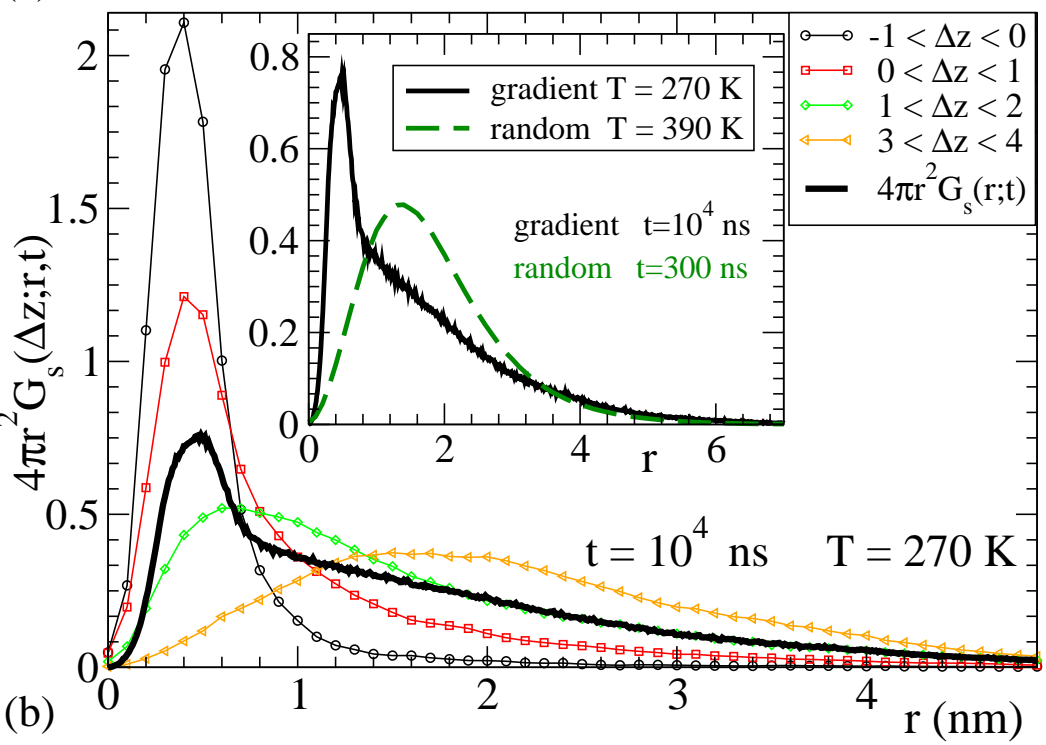

FIG. 12: Main panels: van Hove self-correlation function (symbols) $4 \pi r^{2} G_{\mathrm{s}}(\Delta z ; r, t)$ for monomers, in the gradient copolymer, initially located at a distance $\Delta z$ from the nearest interface (see legend). (a): Data for the F-monomers in the bead-spring model at $T=0.14$ and time $t=2 \times 10^{5}$. (b): Data for the AN-monomers in the polyester at $T=270 \mathrm{~K}$ and $t=10^{4} \mathrm{~ns}$. The solid lines in both main panels represent the corresponding global functions $4 \pi r^{2} G_{\mathrm{s}}(r, t)$. Insets: Comparison beween the global functions in the gradient and random copolymers, at selected times and temperatures with the same $\left\langle\Delta r_{\alpha}^{2}(t)\right\rangle \sim 4$. 

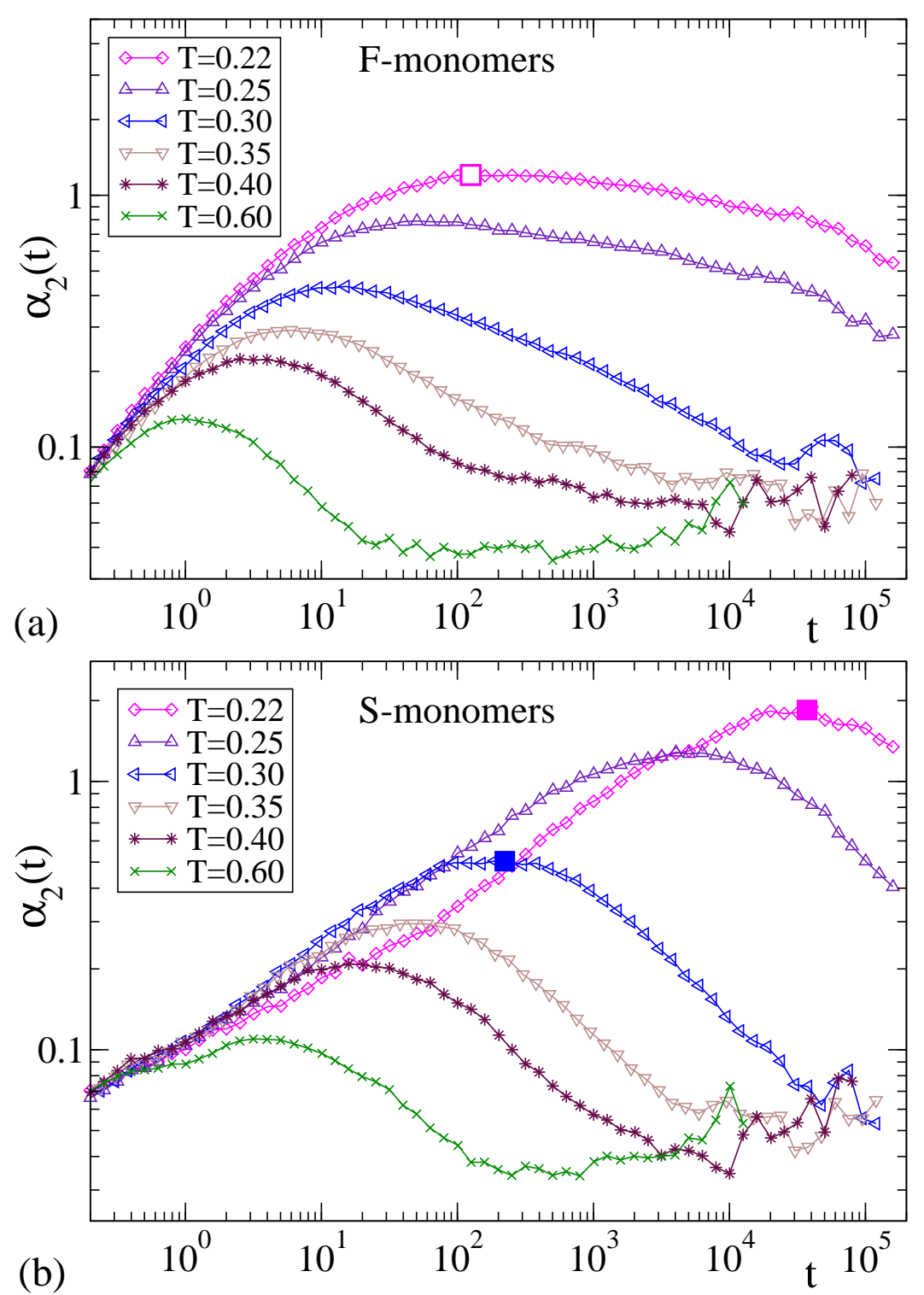

FIG. 13: Non-gaussian parameter in the bead-spring random copolymer, at several temperatures (see legend). (a): F-monomers. (b): S-monomers. Big squares indicate the maxima for selected data sets, and correspond to the same times, temperatures, and monomer species of their counterparts (with same symbol codes) in Fig. 8a. 

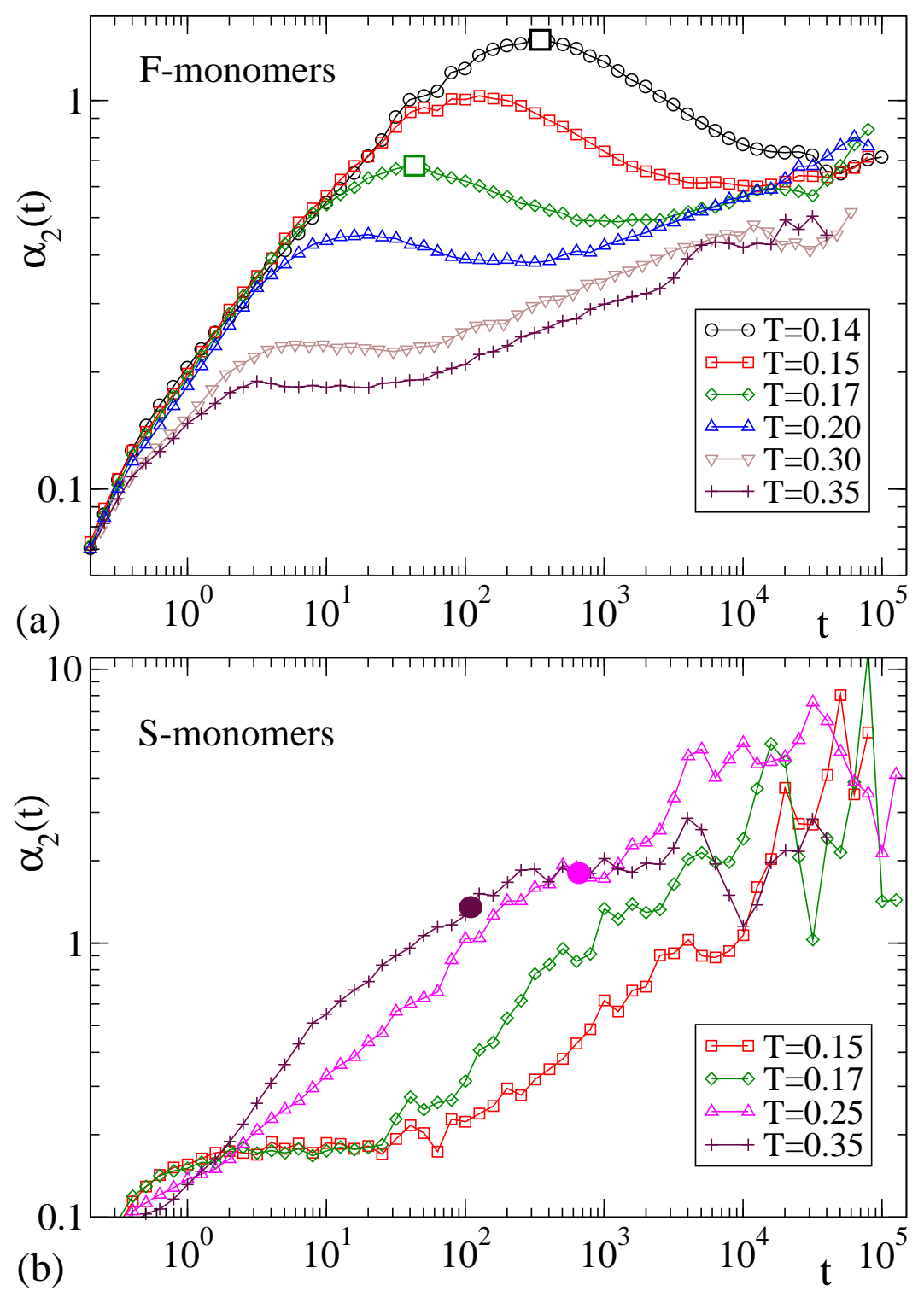

FIG. 14: Non-gaussian parameter in the bead-spring gradient copolymer, at several temperatures (see legend). (a): F-monomers. (b): S-monomers. Big symbols for selected data sets correspond to the same times, temperatures, and monomer species of their counterparts (with same symbol codes) in Fig. 10a. 

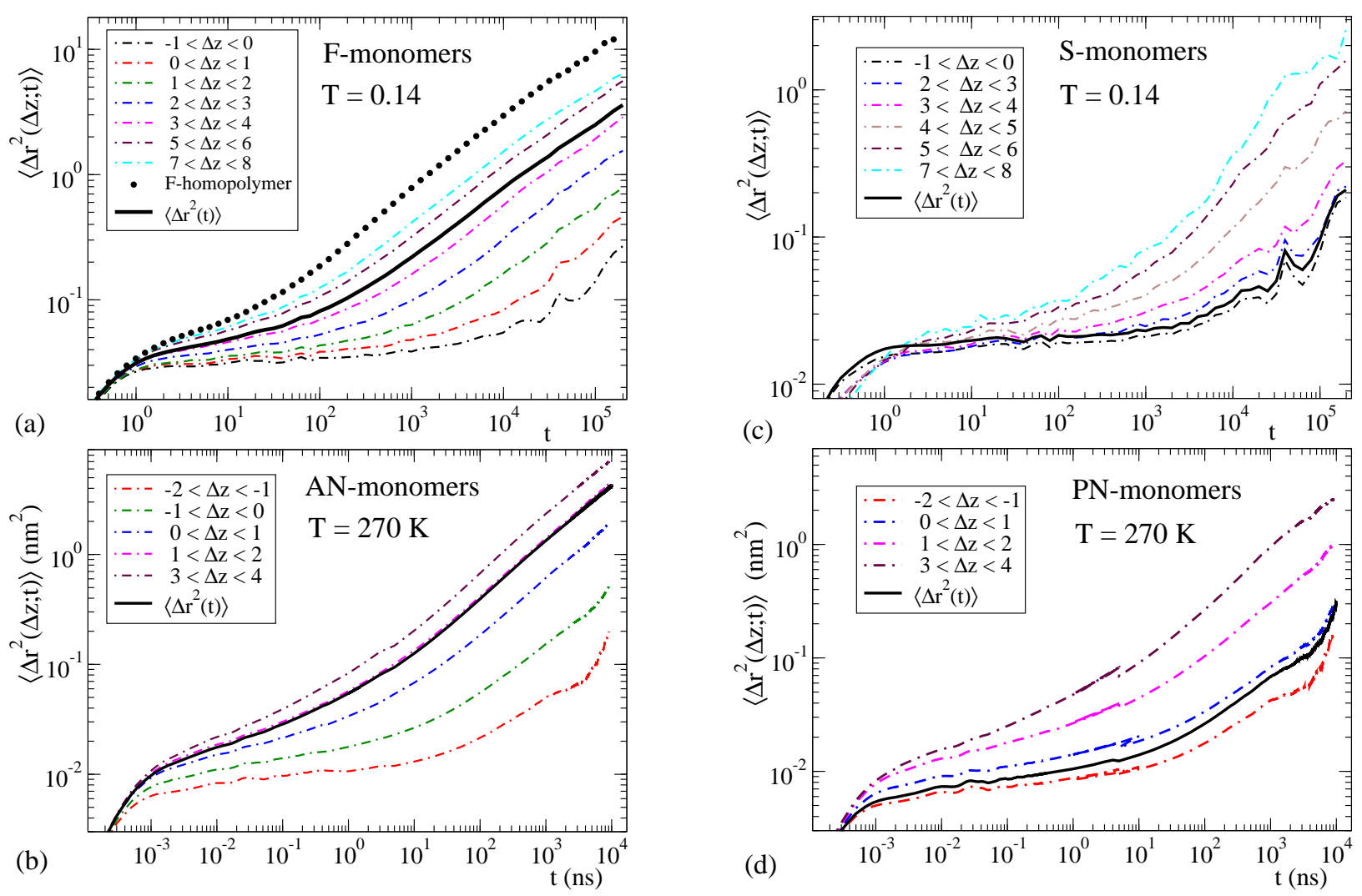

FIG. 15: Dashed-dotted lines: MSD for monomers in the gradient copolymer initially located at a distance $\Delta z$ from the nearest interface (see legend). (a): Data for the F-monomers in the beadspring model at $T=0.14$. (b): Data for the AN-units in the polyester at $T=270 \mathrm{~K}$. (c): Data for the S-monomers in the bead-spring model at $T=0.14$. (d): Data for the PN-units in the polyester at $T=270 \mathrm{~K}$. The solid lines in all panels represent the corresponding global MSD, $\left\langle\Delta r^{2}(t)\right\rangle$. Panel (a) includes data of the F-homopolymer for comparison (symbols). 

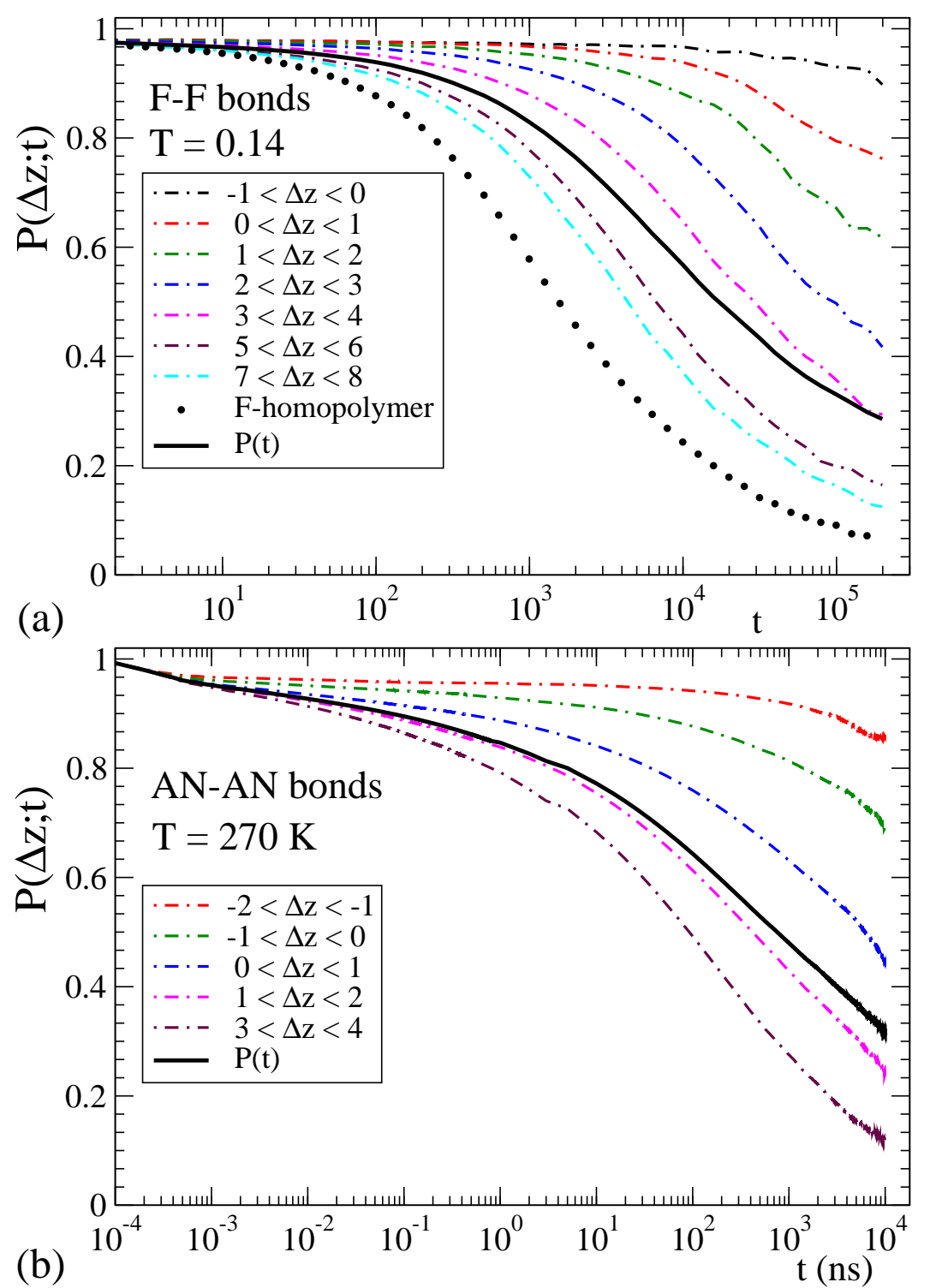

FIG. 16: Dashed-dotted lines: orientational correlator for bonds in the gradient copolymer initially located at a distance $\Delta z$ from the nearest interface (see legend). (a): Data for the F-F bonds in the bead-spring model at $T=0.14$. (b): Data for the AN-AN bonds in the polyester at $T=270 \mathrm{~K}$. The solid lines in both panels represent the corresponding global correlator $P(t)$. Panel (a) includes data of the F-homopolymer for comparison (symbols). 

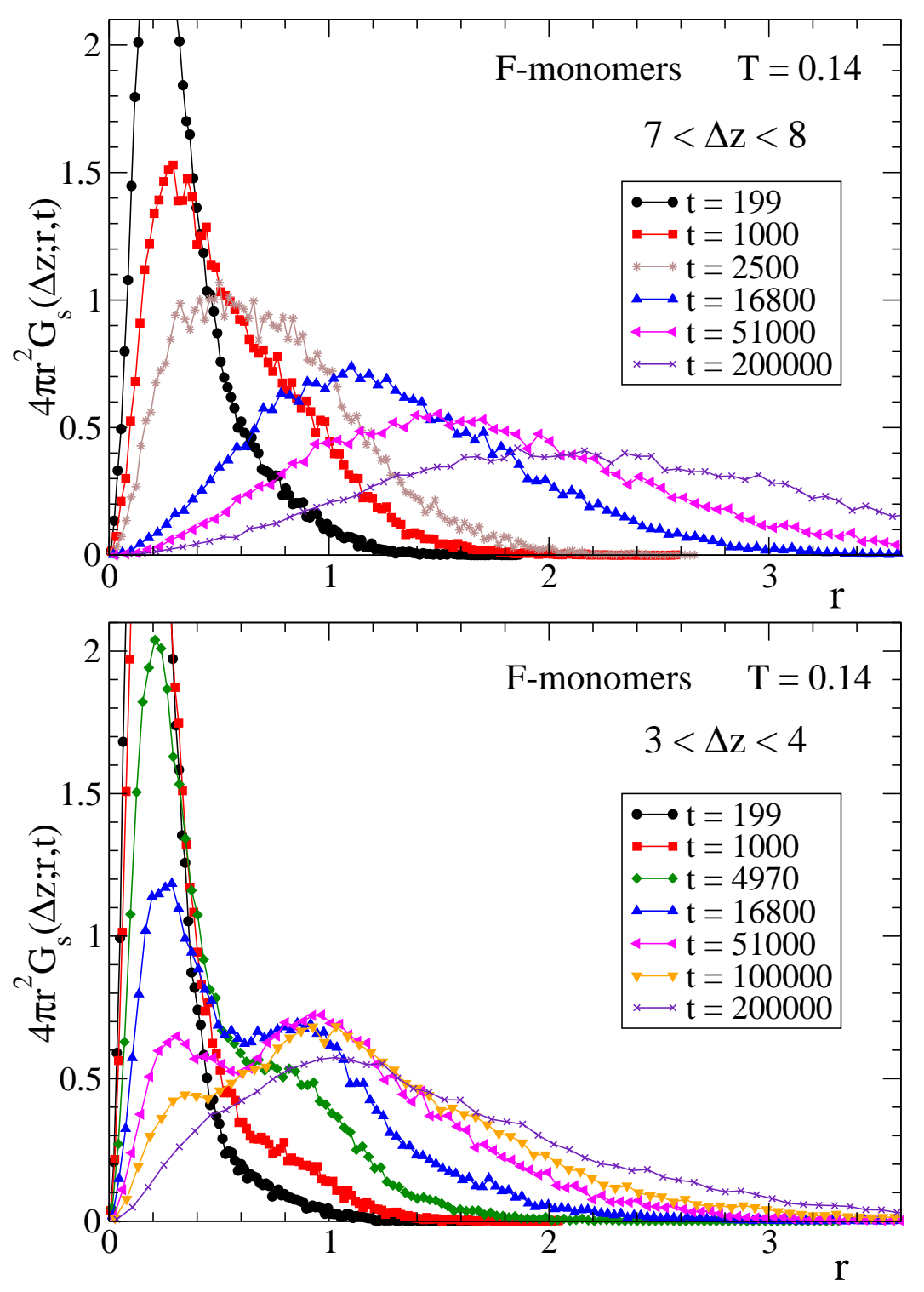

FIG. 17: Slab-dependent van Hove self-correlations functions for the F-monomers in the gradient copolymer at $T=0.14$. Data are shown for two specific slabs, $7<\Delta z<8$ (a) and $3<\Delta z<4$ (b). The selected times are given in the legend. 

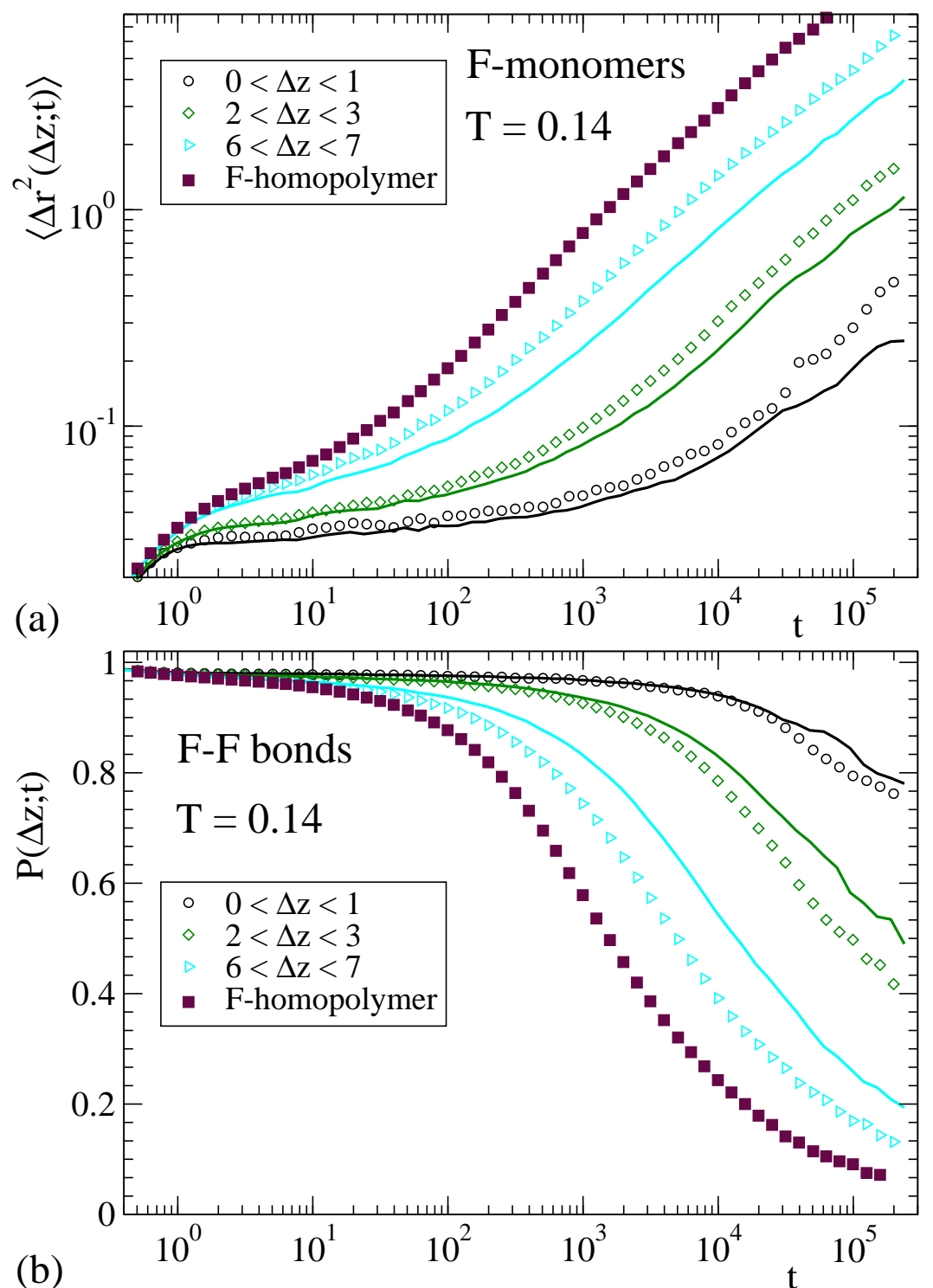

FIG. 18: (a): Slab-dependent MSD (a) and bond correlator (b), of the F-component in the beadspring gradient copolymer at $T=0.14$. In both panels lines and empty symbols correspond to gradient profiles with $C_{0}=1.2$ (more weakly segregated) and $C_{0}=1.8$ (more strongly segregated), respectively. Data sets with same colours correspond to the same $\Delta z$ (see legend). Results for the F-homopolymer at the same $T$ are included for comparison (filled squares). 

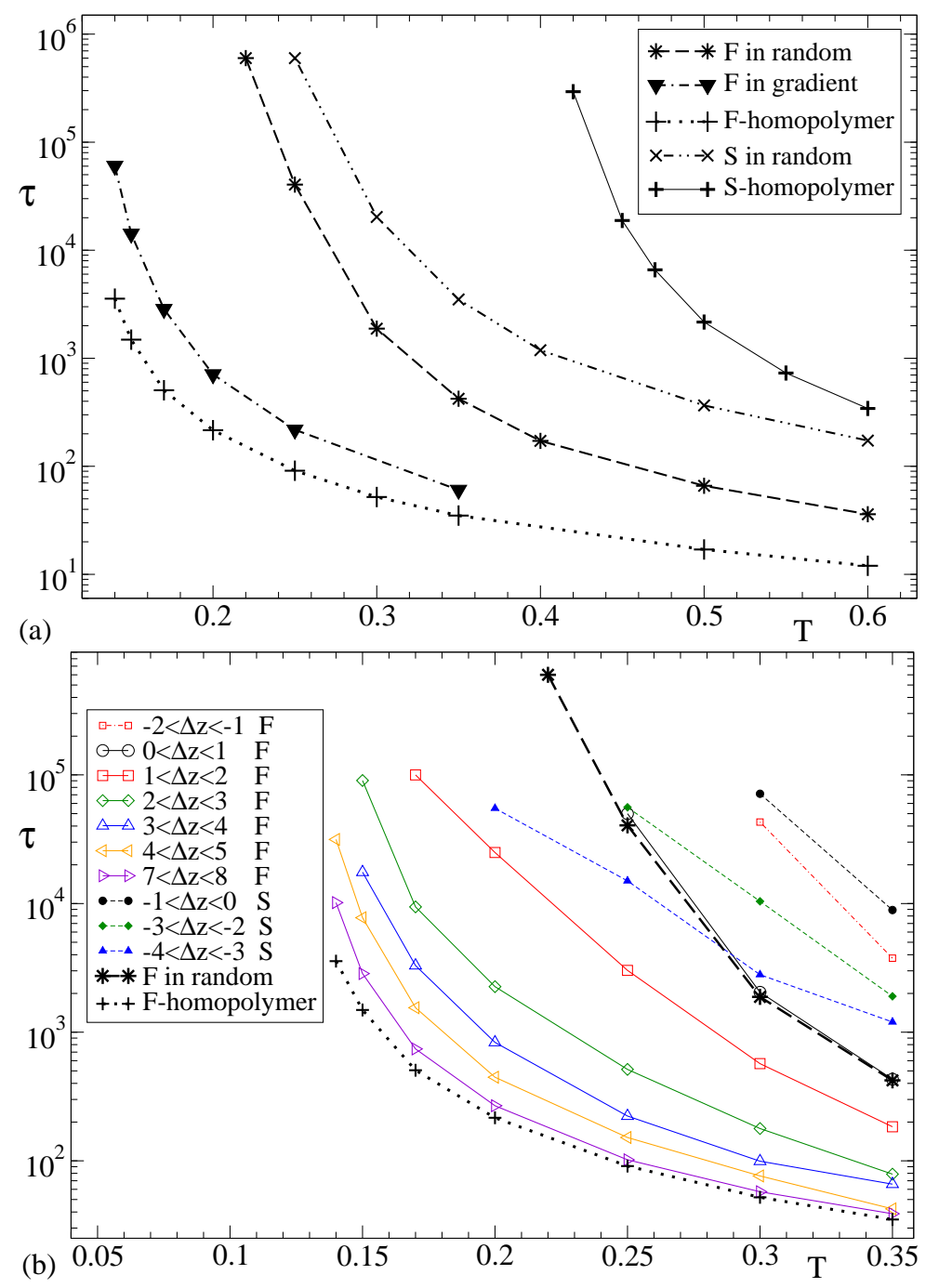

FIG. 19: (a): Temperature dependence of the relaxation times for the global correlators $P(t)$ of the F-F and S-S bonds in the bead-spring systems. Results are given for the gradient and random copolymers, as well as for the homopolymers (see legend). (b): Temperature dependence of the slab-dependent relaxation times for F-F (empty symbols) and S-S bonds (filled symbols) in the bead-spring gradient copolymer. Positive values of $\Delta z$ (big empty symbols) correspond to bonds initially located in domains rich in their same species. Negative values (small symbols) correspond to those located in domains rich in the other species. For comparison we also include data from panel (a) for global relaxation times for F-F (big stars) and S-S bonds (big crosses) in the random copolymer, as well as for the F-homopolymer (big pluses). Lines in all data sets of both panels are guides for the eyes. 
TABLE OF CONTENTS GRAPHIC
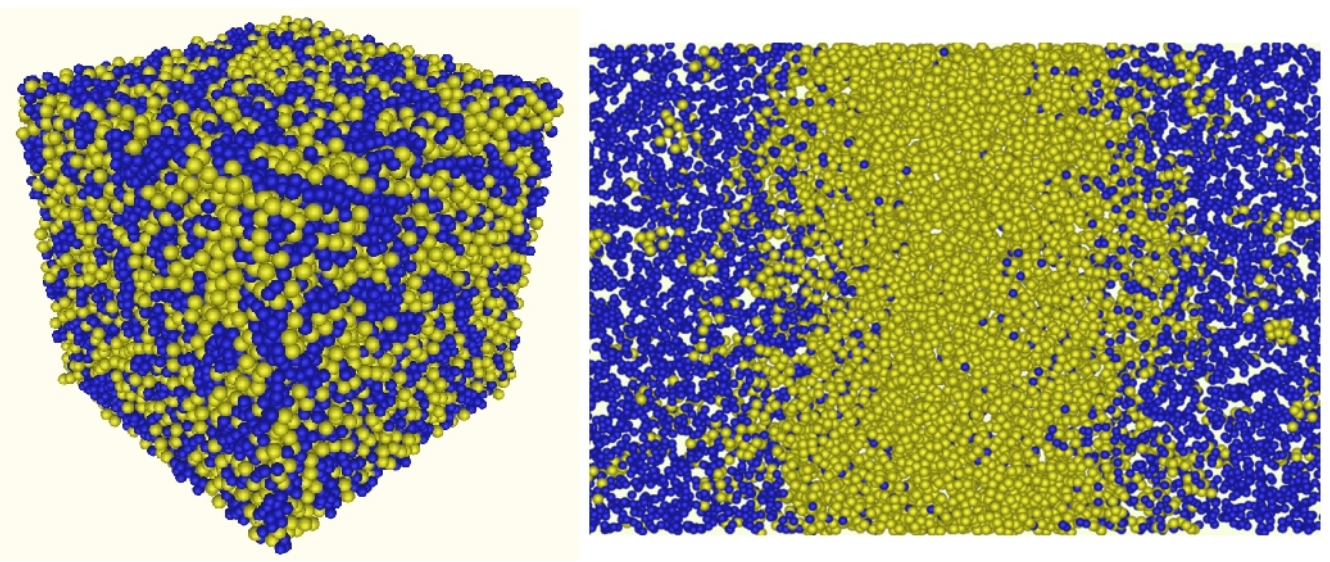

Dynamic heterogeneity in random and gradient copolymers: A computational investigation

Mohammed Zakaria Slimani, Angel J. Moreno, Giulia Rossi and Juan Colmenero 